\title{
Supplementary Material-Spatial and temporal analysis of extreme sea level and skew surge events around the coastline of New Zealand
}

Scott A. Stephens ${ }^{1}$, Robert G. Bell ${ }^{1}$, Ivan Haigh ${ }^{2}$

${ }^{1}$ National Institute of Water and Atmospheric Research, PO Box 11 115, Hamilton 3251, New Zealand

$5 \quad{ }^{2}$ Ocean and Earth Science, National Oceanography Centre, University of Southampton, European Way, Southampton, SO14 $3 \mathrm{ZH}, \mathrm{UK}$ 

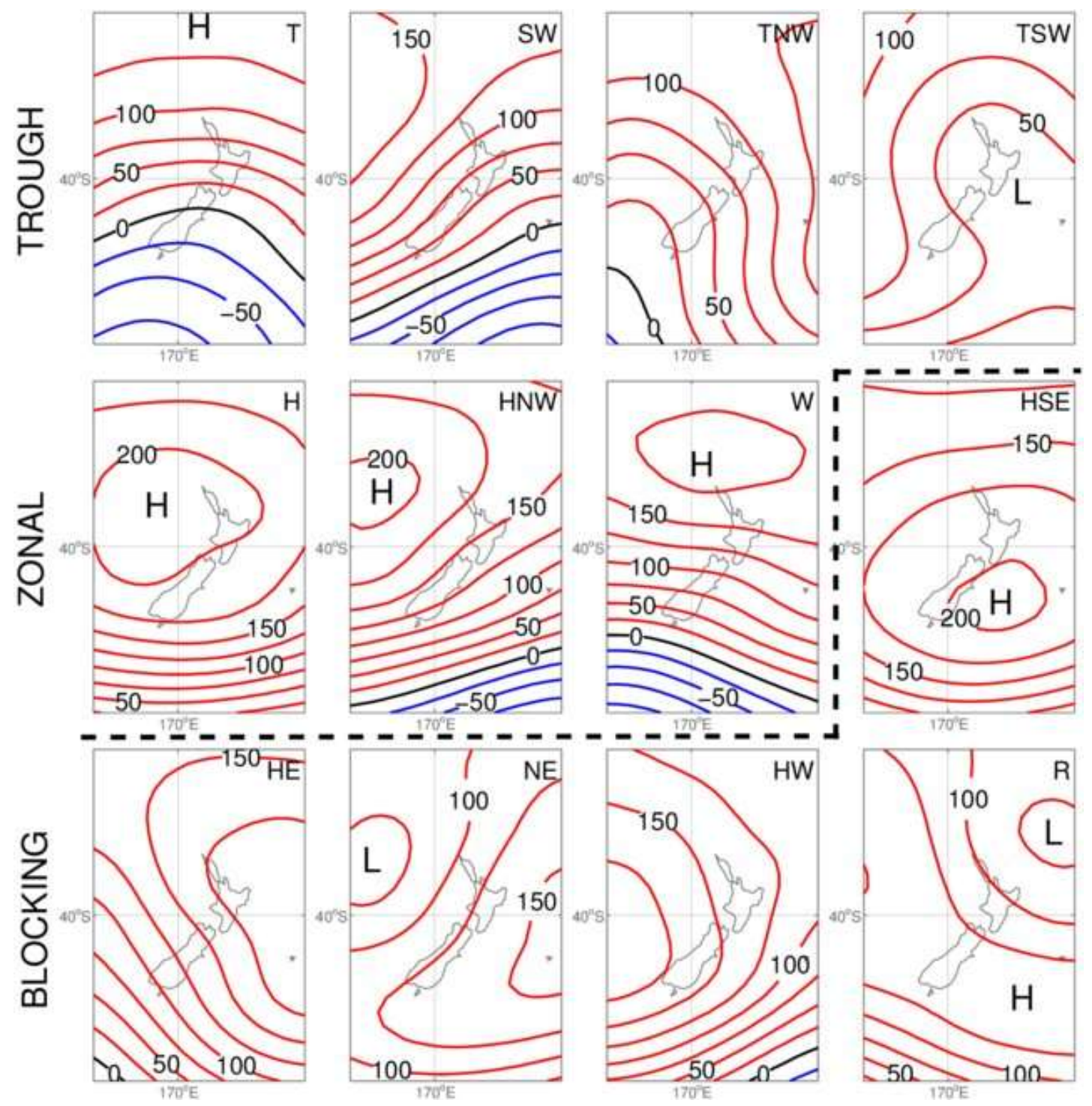

10 Figure S1. The twelve Kidson weather types, shown as average patterns of $1000 \mathrm{hPa}$ geopotential height (analogous to mean sea-level pressure). Names for the types are indicated in the top right of each panel. Source: Ackerley et al. (2011). The three regimes are indicated at the left: the top row is the trough regime, the first three in the second row are the zonal regime, and the rest form the blocking regime. See Kidson (2000) for further details. 

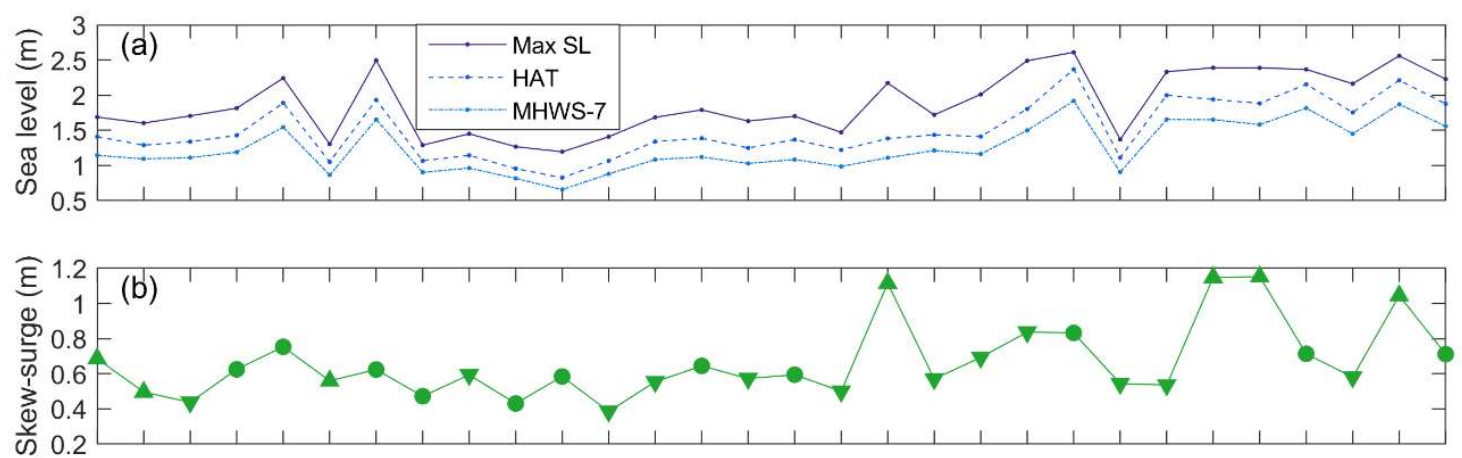

ल

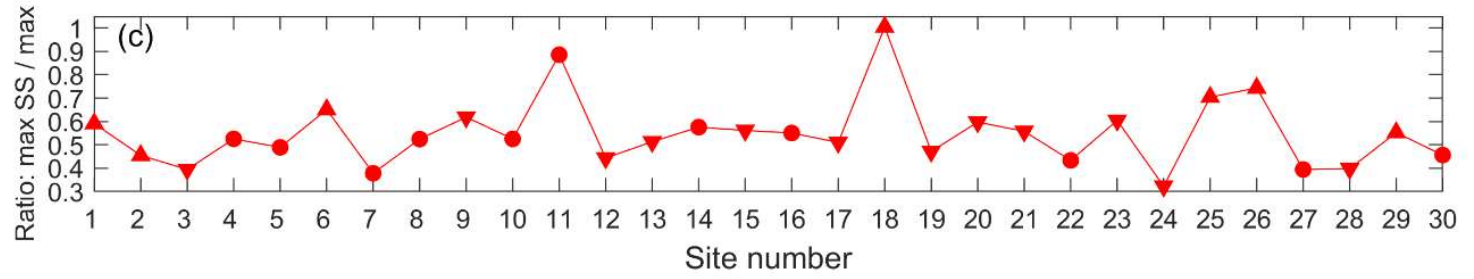

Figure S2. (a) Maximum sea level; (b) maximum skew-surge; and (c) the ratio of maximum skew-surge to sea level; plotted relative to the distance clockwise around the coast of $\mathrm{NZ}$ from the tip of the North Island. Upward-pointing triangles represent small enclosed estuaries, circles represent large harbours or those with wide entrances and downward-pointing triangles represent the open coast. 
Height of sea-level components $(\mathrm{m})$ within high-water percentile bands at Auckland
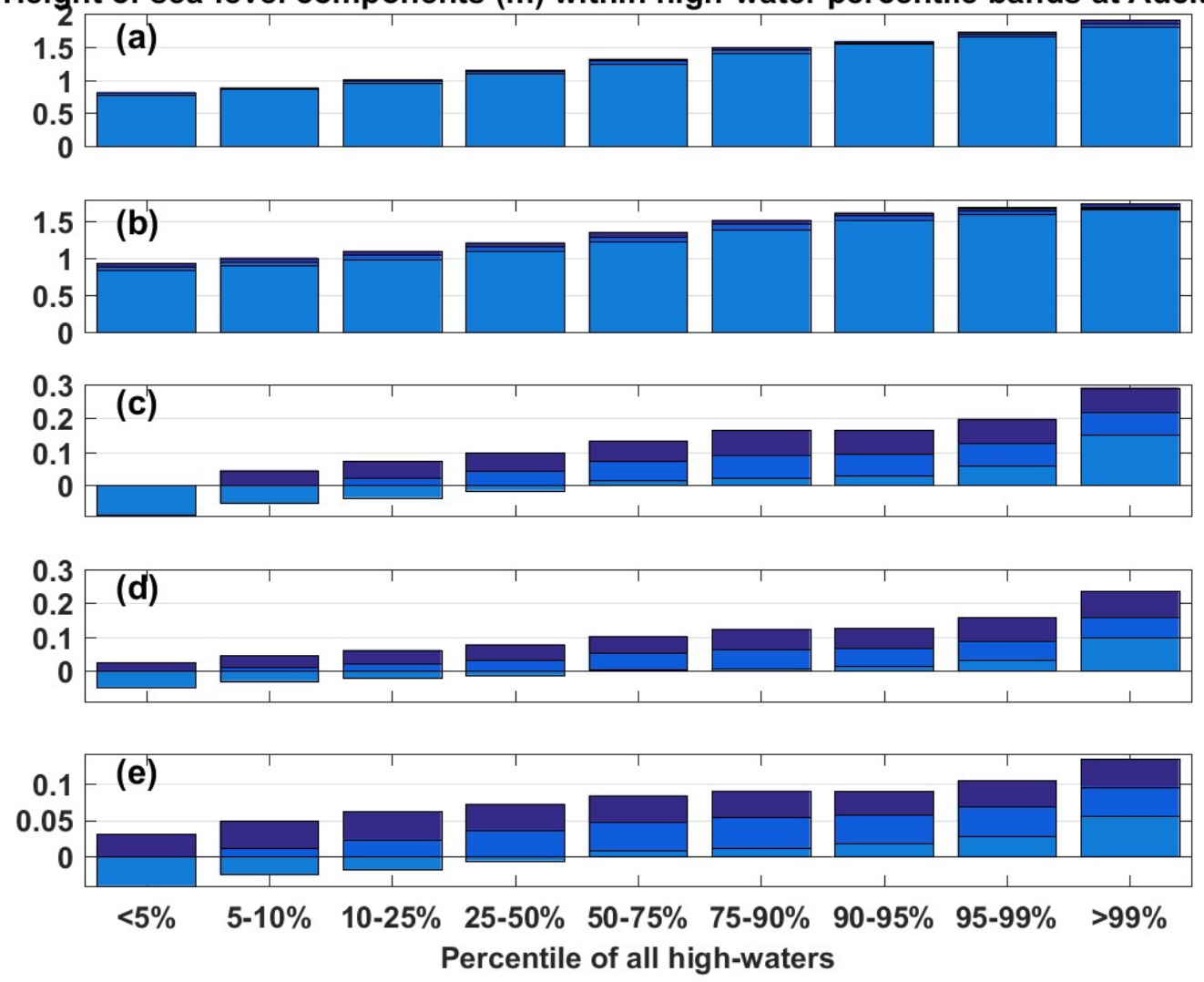

Figure S3. Heights of sea-level components (m) within high-water percentile bands at Auckland NZ. (a) sea-level at high-water (b) hightide (c) skew-surge (d) skew-surge minus mean sea-level anomaly (MSLA) (e) MSLA. The coloured bars from light to dark respectively mark the height of the 50th, 75th and 90th percentile of the sea-level components that match the high-waters within the percentile bands along the horizontal axis. The general pattern of behaviour shown here for Auckland is seen everywhere in NZ. 
Percentile of sea-level components relative to high-water within high-water percentile bands: NZ mean
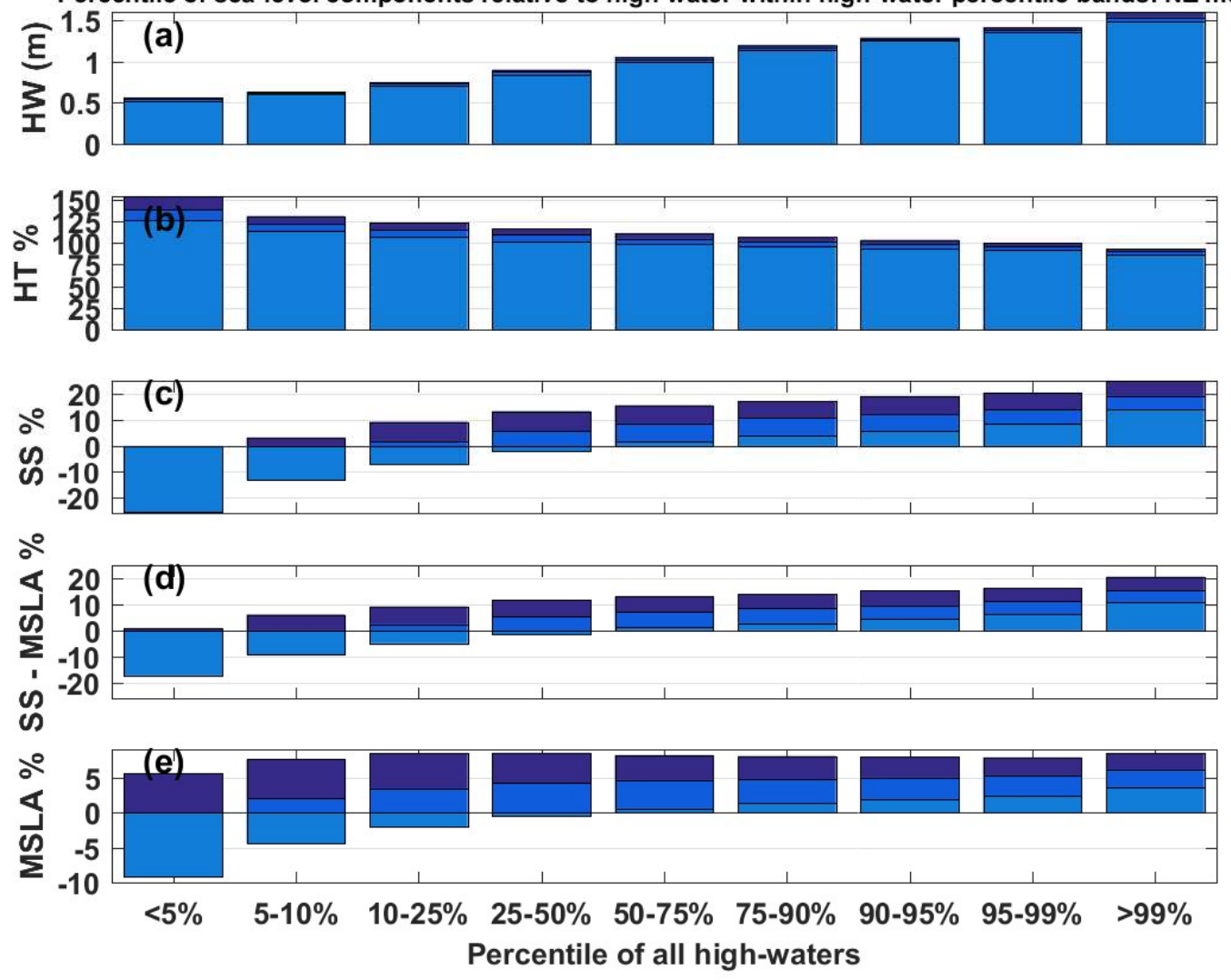

Figure S4. Percentile of sea-level component height relative to high-water height within high-water percentile bands-NZ mean. (a) high-water height (m) (b) high-tide (c) skew-surge (d) skew-surge minus mean sea-level anomaly (MSLA) (e) MSLA. The coloured bars from light to dark respectively mark the height of the 50th, 75th and 90th percentile of the sea-level components that match the highwaters within the percentile bands along the horizontal axis. The mean patterns reflect those at individual sites. 

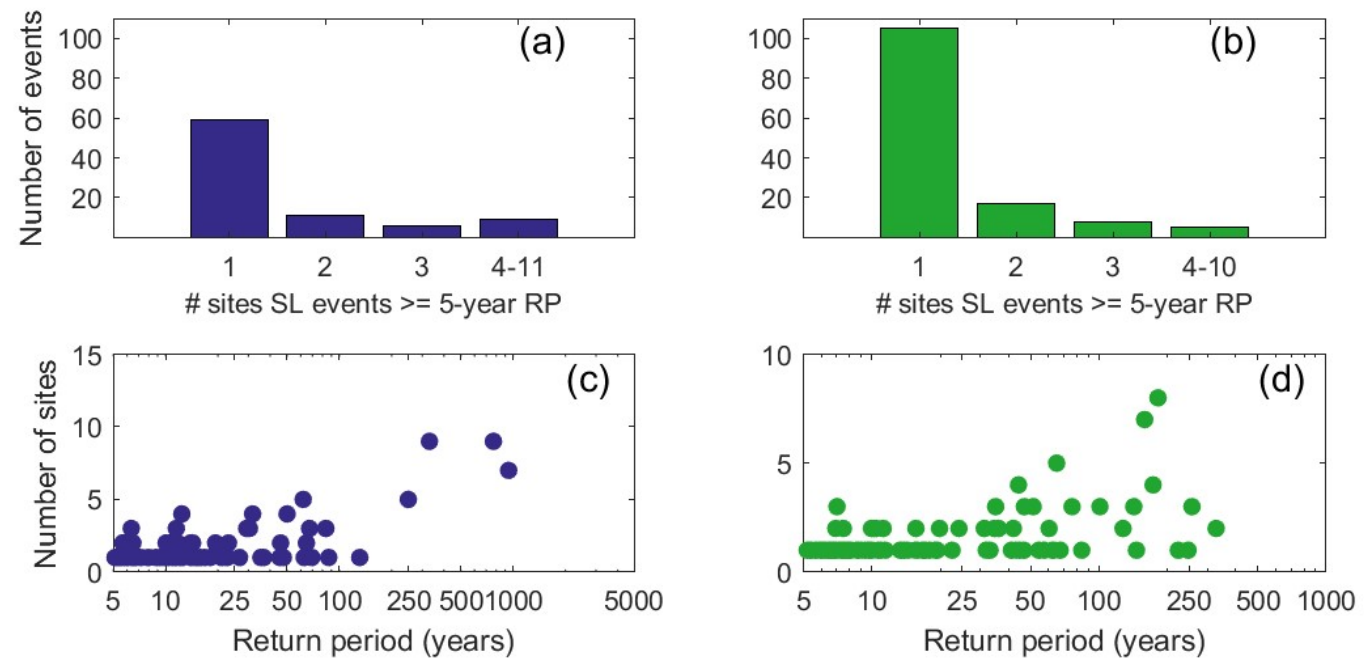

Figure S5. Histograms showing the number of sites where the 1 in 5-year return level was reached or exceeded for (a) extreme sea-level events and (b) skew-surge events. The offset return period of the (c) highest sea levels in each of the 155 extreme sea-level observations, and (d) the highest skew-surge return periods for each of 191 skew-surge observations, plotted against the number of sites where the 1 in 5-year return period was reached or exceeded during an event.

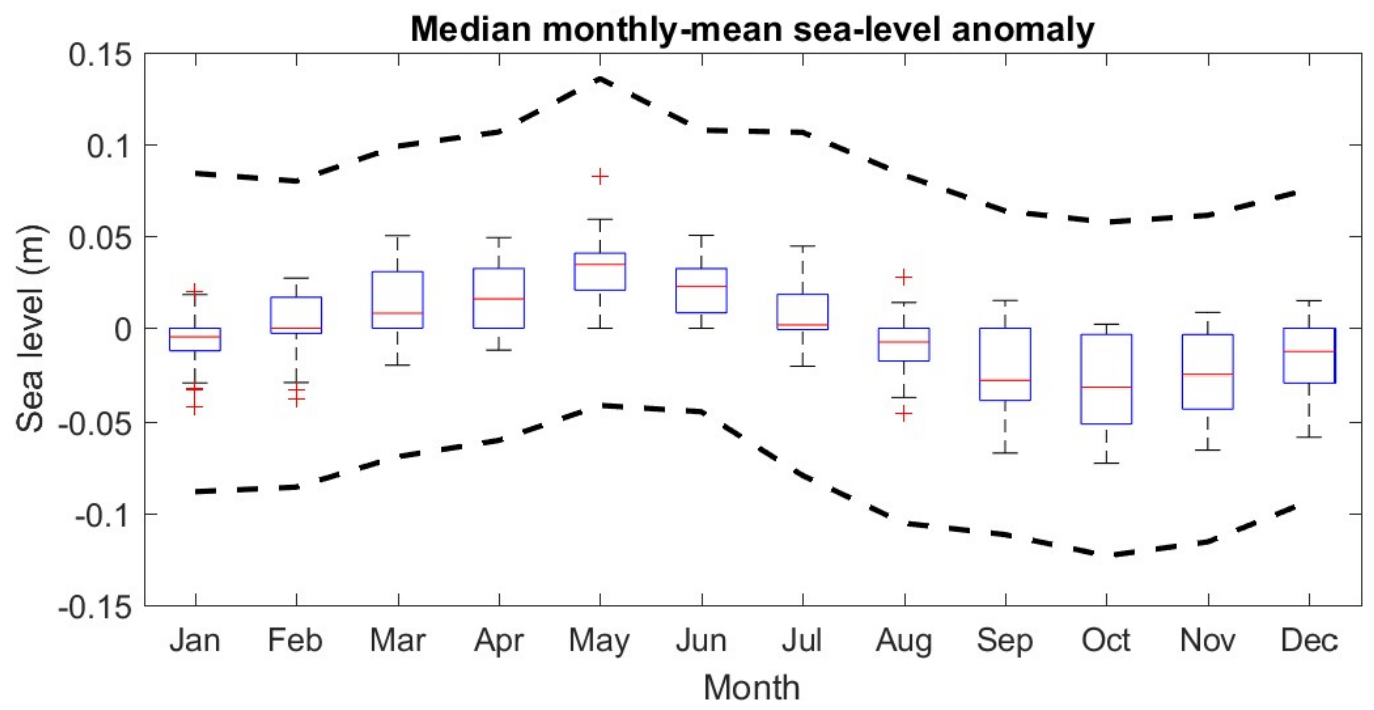

Figure S6. Median (all 30 sites) monthly-mean sea-level anomaly (MSLA). Red line marks median, blue box marks interquartile range (25-75\%), whiskers mark 2.7-99.3\% coverage for normally-distributed data and red crosses mark outliers beyond the whisker range. 

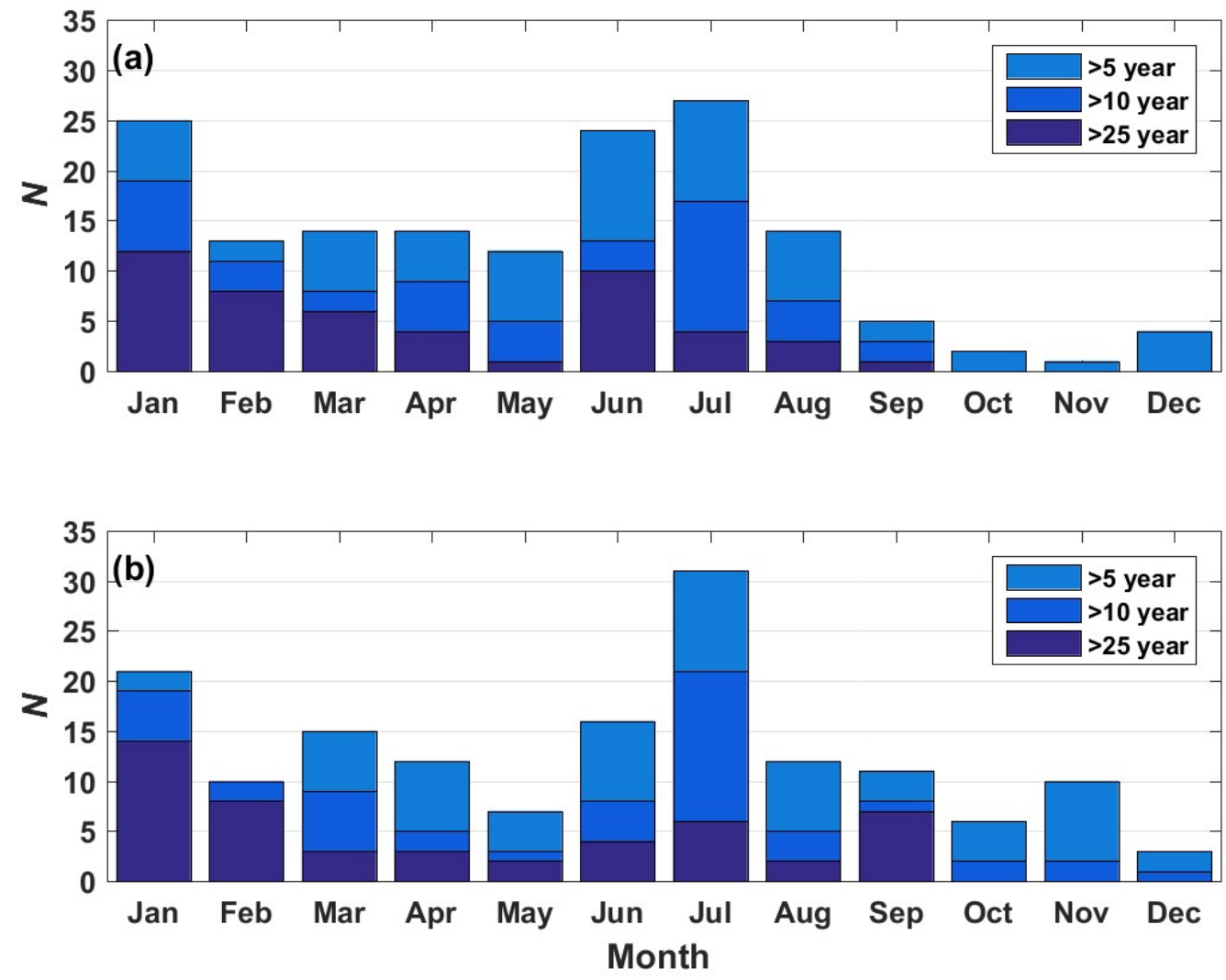

Figure S7. Seasonal distribution of extreme sea-level events. (a) Extreme Sea Level (b) Extreme Sea Level after first removing MSLA. $N=$ number of occasions. 

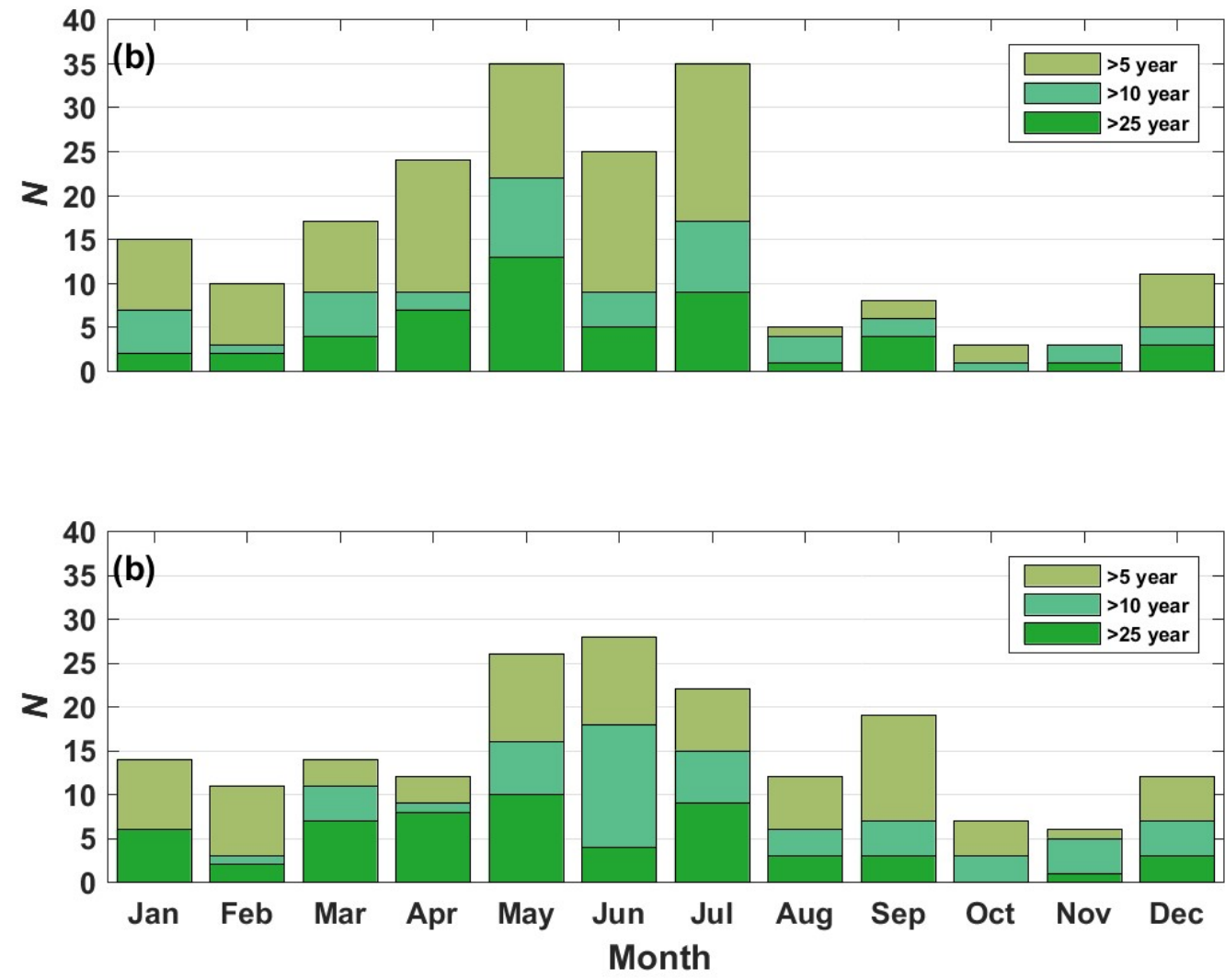

Figure S8. Seasonal distribution of extreme skew-surge events. (a) extreme skew-surge (b) extreme skew-surge after first removing MSLA. $N=$ number of occasions. 


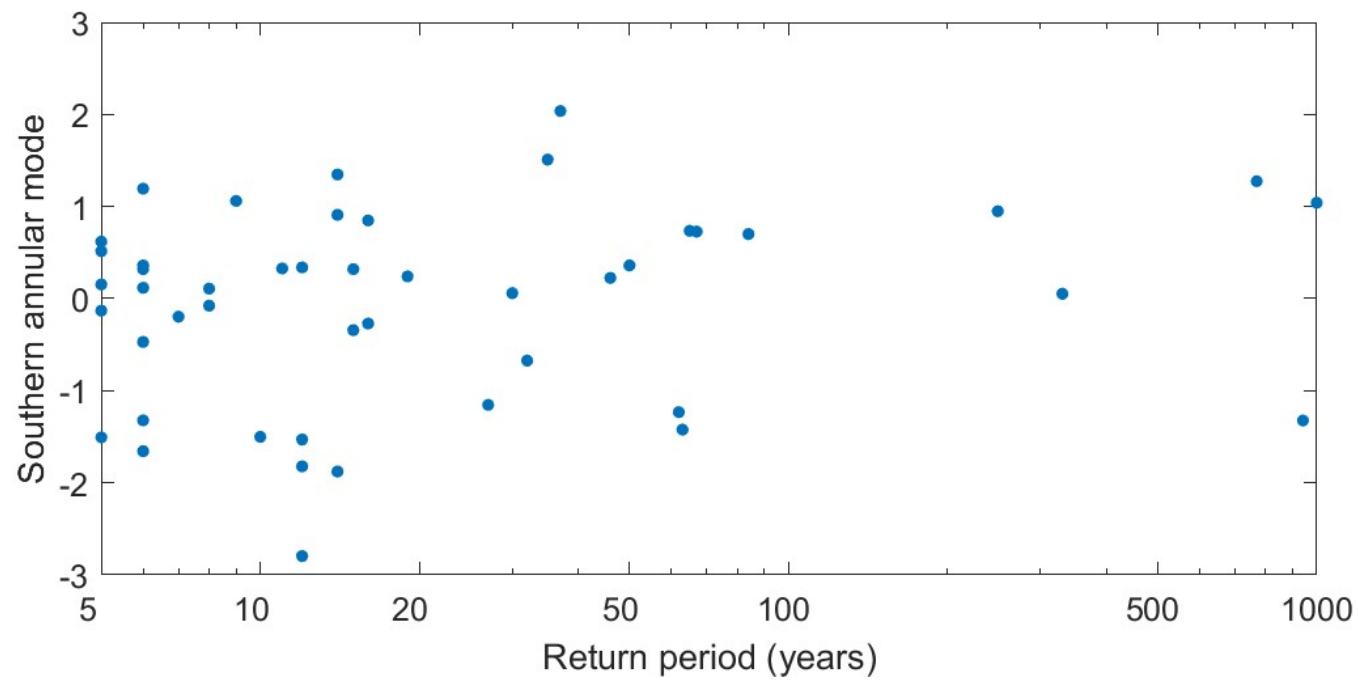

Figure S9. Extreme sea-level return period versus Southern Annular Mode (SAM) climate index.

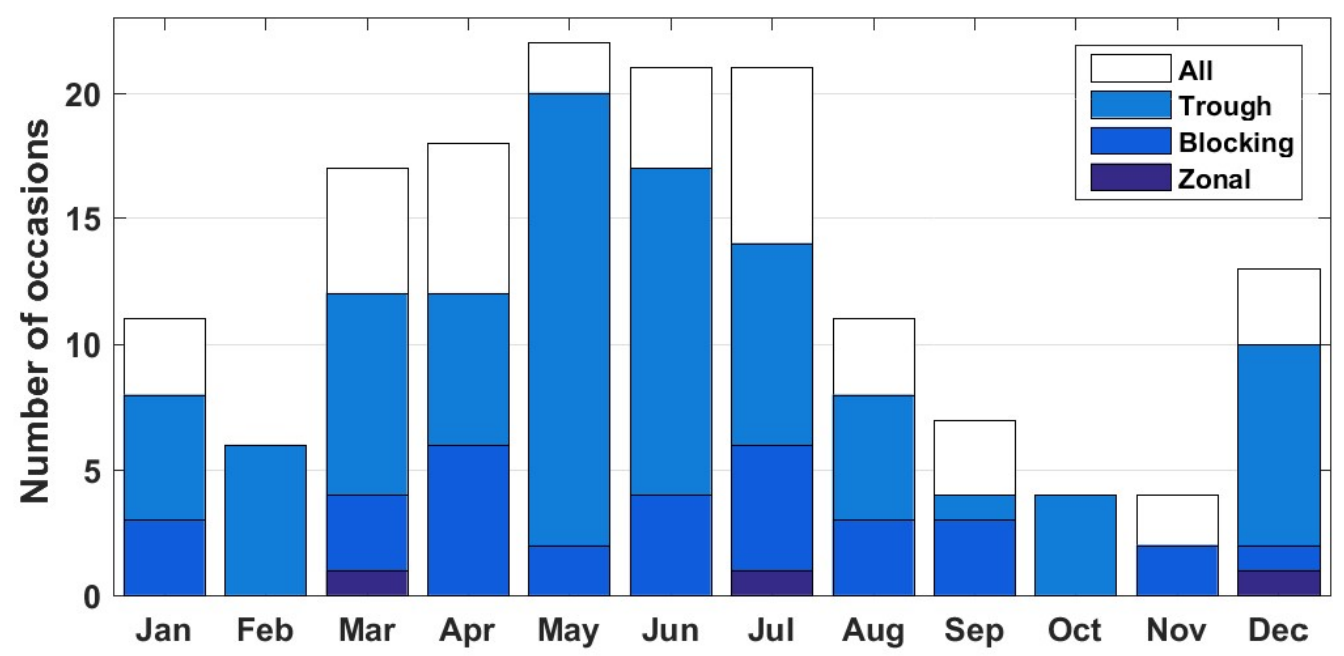

Figure S10. Kidson (2000) weather type associated with unique storm events that contributed to $\geq$ 5-year return level sea-level or skewsurge. Kidson types are available for 155 storm events since 1948, so the occurrence distribution shown here differs from that in Figure 7 which covers 191 storm events with effects observed in any of the sea-level gauge records. [Selected $\geq 5$-year return level sea-level and skew-surge events including MSLAJ. 


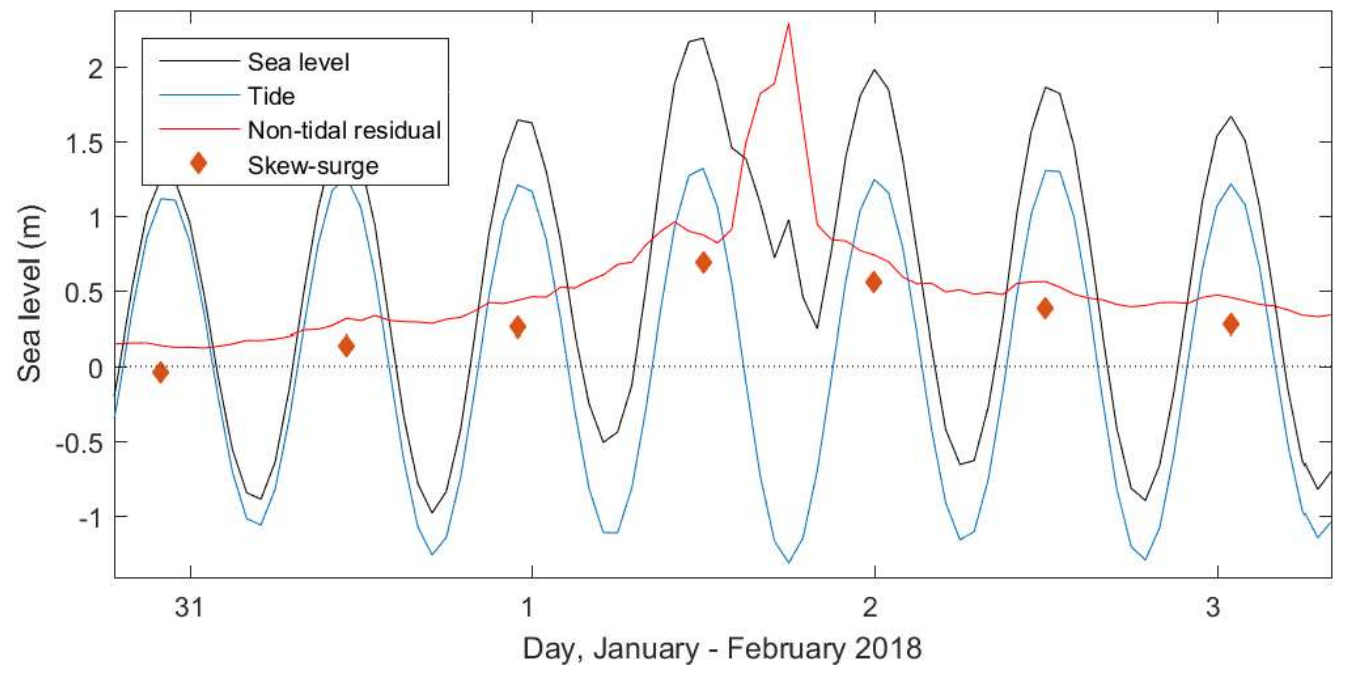

60 Figure S11. Sea level at Jackson Bay (site 20; Figure 1a) during ex-tropical cyclone Fehi. 
Supplementary Tables

65

Table S1. Names and locations of study sites.

\begin{tabular}{|c|c|c|c|c|c|c|}
\hline $\begin{array}{c}\text { Site } \\
\text { Number }\end{array}$ & Site Name & $\begin{array}{c}\text { Longitude } \\
\text { (degrees) }\end{array}$ & $\begin{array}{l}\text { Latitude } \\
\text { (degrees) }\end{array}$ & Range & $\begin{array}{c}\text { Number of } \\
\text { years } \\
\text { (range) }\end{array}$ & $\begin{array}{c}\text { Number of } \\
\text { years } \\
\geq 75 \% \\
\text { complete } \\
\text { data }\end{array}$ \\
\hline 1 & Rangaunu & 173.26 & -35.01 & 2004-2014 & 10 & 9 \\
\hline 2 & Whangaroa & 173.74 & -35.05 & $2008-2014$ & 6 & 5 \\
\hline 3 & Opua & 174.12 & -35.31 & 1990-2014 & 24 & 22 \\
\hline 4 & Marsden Point & 174.50 & -35.84 & 1964-2018 & 55 & 43 \\
\hline 5 & Auckland & 174.77 & -36.84 & 1903-2018 & 115 & 106 \\
\hline 6 & Whitianga & 175.71 & -36.83 & 1999-2018 & 19 & 19 \\
\hline 7 & Tararu & 175.52 & -37.13 & 1990-2018 & 28 & 27 \\
\hline 8 & Tauranga & 176.18 & -37.64 & 1989-2016 & 27 & 25 \\
\hline 9 & Moturiki & 176.19 & -37.63 & 1974-2018 & 44 & 42 \\
\hline 10 & Gisborne & 178.03 & -38.67 & $2004-2013$ & 9 & 7 \\
\hline 11 & Wellington & 174.78 & -41.28 & 1944-2018 & 74 & 71 \\
\hline 12 & Kaikoura & 173.70 & -42.42 & 1994-2007 & 13 & 12 \\
\hline 13 & Sumner & 172.57 & -43.57 & 1994-2018 & 24 & 22 \\
\hline 14 & Lyttelton & 172.72 & -43.61 & 1924-2018 & 95 & 81 \\
\hline 15 & Timaru & 171.25 & -44.39 & 2001-2018 & 17 & 17 \\
\hline 16 & Dunedin & 170.51 & -45.88 & 1899-2013 & 114 & 85 \\
\hline 17 & Green Island & 170.38 & -45.96 & $2002-2018$ & 16 & 16 \\
\hline 18 & Invercargill & 168.33 & -46.42 & 1992-2018 & 26 & 26 \\
\hline 19 & Dog Island & 168.41 & -46.65 & $1997-2018$ & 21 & 21 \\
\hline 20 & Jackson Bay & 168.62 & -43.96 & 1998-2018 & 20 & 15 \\
\hline 21 & Charleston & 171.43 & -41.91 & $1997-2018$ & 21 & 15 \\
\hline 22 & Nelson & 173.27 & -41.26 & 1984-2018 & 34 & 20 \\
\hline 23 & Kapiti & 174.94 & -40.84 & $1997-2012$ & 15 & 14 \\
\hline 24 & Taranaki & 174.03 & -39.06 & 1984-2018 & 34 & 28 \\
\hline
\end{tabular}




\begin{tabular}{|l|l|c|c|c|c|c|}
\hline 25 & Kawhia & 174.82 & -38.07 & $2008-2018$ & 10 & 9 \\
\hline 26 & Raglan & 174.88 & -37.79 & $2008-2018$ & 10 & 6 \\
\hline 27 & Onehunga & 174.52 & -37.05 & $2001-2018$ & 18 & 16 \\
\hline 28 & Anawhata & 174.45 & -36.93 & $1999-2011$ & 13 & 13 \\
\hline 29 & Helensville & 174.45 & -36.67 & $2005-2014$ & 9 & 8 \\
\hline 30 & Pouto Point & 174.18 & -36.36 & $2002-2018$ & 17 & 14 \\
\hline
\end{tabular}


Table S2. Extreme sea-level events. For completeness the inferred extreme sea-level 4 May 1938 at Tararu is included (Stephens 2018).

\begin{tabular}{|c|c|c|c|c|c|c|c|c|}
\hline 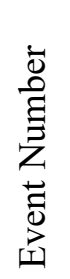 & 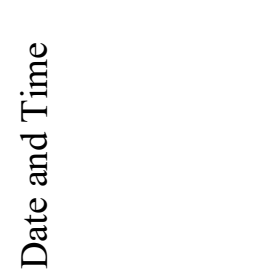 & 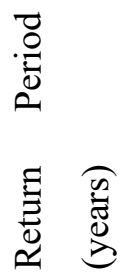 & 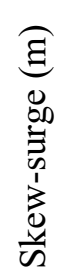 & 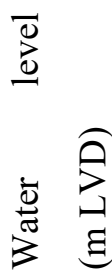 & 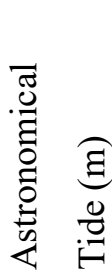 & $\begin{array}{l}\underset{\Xi}{\leftrightarrows} \\
\underset{\mathscr{S}}{\Sigma}\end{array}$ & 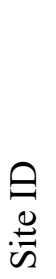 & 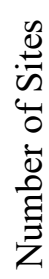 \\
\hline 1 & $1 / 02 / 201811: 00$ & $>1000$ & 0.84 & 2.49 & 1.65 & -0.02 & 21 & 9 \\
\hline 2 & 15/06/1999 18:00 & 942 & 0.36 & 1.41 & 1.05 & 0.10 & 12 & 7 \\
\hline 3 & $5 / 01 / 2018$ 10:00 & 770 & 0.62 & 2.50 & 1.87 & 0.04 & 7 & 9 \\
\hline 4 & 23/01/2011 10:00 & 330 & 0.42 & 1.71 & 1.29 & 0.12 & 3 & 9 \\
\hline 5 & 17/04/1999 10:00 & 249 & 0.58 & 2.16 & 1.58 & 0.10 & 28 & 5 \\
\hline \multirow[t]{2}{*}{6} & 26/03/1936 09:00 & 131 & 0.59 & 2.24 & 1.66 & 0.00 & 5 & 1 \\
\hline & $\begin{array}{l}04 / 05 / 1938 \\
\text { inferred at Tararu }\end{array}$ & $\sim 120$ & 1.26 & 3.00 & 1.74 & 0 & 7 & 1 \\
\hline 7 & $17 / 04 / 1972$ 19:00 & 87 & 0.44 & 1.20 & 0.75 & 0.14 & 11 & 1 \\
\hline 8 & 11/03/1997 22:00 & 84 & 0.52 & 1.81 & 1.29 & 0.17 & 4 & 3 \\
\hline 9 & 4/08/1921 15:00 & 69 & 0.45 & 1.70 & 1.25 & 0.00 & 16 & 1 \\
\hline 10 & 21/07/2017 13:00 & 67 & 0.57 & 1.63 & 1.06 & 0.00 & 15 & 3 \\
\hline 11 & 30/03/1998 03:00 & 65 & 0.89 & 2.17 & 1.28 & 0.22 & 18 & 2 \\
\hline 12 & $16 / 03 / 198015: 00$ & 63 & 0.56 & 1.70 & 1.14 & 0.04 & 16 & 1 \\
\hline 13 & 23/07/2009 23:00 & 62 & 0.40 & 1.36 & 0.97 & 0.03 & 23 & 5 \\
\hline 14 & $26 / 05 / 201314: 00$ & 50 & 0.37 & 1.72 & 1.35 & 0.02 & 19 & 4 \\
\hline 15 & 13/06/1968 22:00 & 48 & 0.62 & 1.77 & 1.16 & 0.05 & 4 & 1 \\
\hline 16 & 16/04/2003 18:00 & 46 & 0.59 & 1.43 & 0.84 & 0.07 & 9 & 2 \\
\hline 17 & 4/02/1904 09:00 & 45 & 0.36 & 2.16 & 1.80 & 0.07 & 5 & 1 \\
\hline 18 & 11/03/2016 03:00 & 37 & 0.93 & 2.11 & 1.18 & 0.03 & 18 & 1 \\
\hline 19 & 13/08/2010 01:00 & 35 & 0.46 & 1.79 & 1.33 & 0.09 & 20 & 1 \\
\hline 20 & 27/01/1940 04:00 & 35 & 0.48 & 1.73 & 1.25 & 0.17 & 14 & 1 \\
\hline 21 & $30 / 08 / 200020: 00$ & 32 & 0.28 & 1.28 & 1.01 & 0.12 & 8 & 4 \\
\hline 22 & $3 / 07 / 200021: 00$ & 30 & 0.33 & 1.74 & 1.41 & 0.09 & 4 & 3 \\
\hline 23 & 14/07/1995 20:00 & 29 & 0.48 & 2.27 & 1.79 & 0.05 & 7 & 3 \\
\hline 24 & 15/06/1991 22:00 & 27 & 0.29 & 1.28 & 0.99 & 0.09 & 8 & 1 \\
\hline
\end{tabular}




\begin{tabular}{|c|c|c|c|c|c|c|c|c|}
\hline 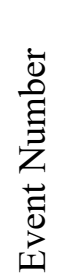 & 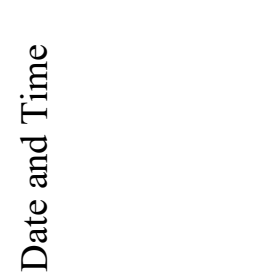 & 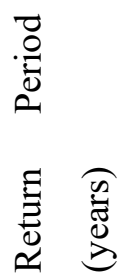 & 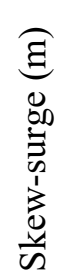 & 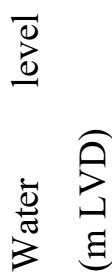 & 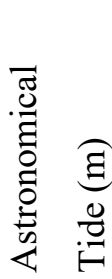 & $\begin{array}{l}\underset{\Xi}{\Xi} \\
\stackrel{\mathscr{S}}{\Sigma}\end{array}$ & 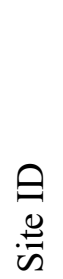 & 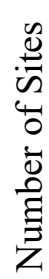 \\
\hline 25 & 20/07/1978 06:00 & 23 & 0.43 & 1.39 & 0.97 & 0.08 & 9 & 2 \\
\hline 26 & 22/06/1978 20:00 & 23 & 0.26 & 1.39 & 1.13 & 0.15 & 9 & 1 \\
\hline 27 & $14 / 06 / 197523: 00$ & 22 & 0.48 & 1.39 & 0.91 & 0.08 & 9 & 1 \\
\hline 28 & 14/02/1918 22:00 & 21 & 0.50 & 2.11 & 1.61 & 0.12 & 5 & 1 \\
\hline 29 & 18/09/2005 23:00 & 19 & 0.43 & 2.23 & 1.80 & -0.01 & 30 & 2 \\
\hline 30 & 27/04/1959 19:00 & 18 & 0.45 & 1.65 & 1.21 & 0.07 & 14 & 1 \\
\hline 31 & $15 / 08 / 193915: 00$ & 17 & 0.44 & 1.69 & 1.26 & 0.13 & 14 & 1 \\
\hline 32 & 21/06/2013 01:00 & 16 & 0.50 & 1.11 & 0.61 & 0.07 & 11 & 1 \\
\hline 33 & $8 / 02 / 198911: 00$ & 16 & 0.37 & 2.49 & 2.12 & 0.15 & 22 & 1 \\
\hline 34 & 27/07/2008 01:00 & 15 & 0.52 & 1.27 & 0.75 & -0.01 & 6 & 1 \\
\hline 35 & 15/08/2018 01:00 & 15 & 0.44 & 2.36 & 1.92 & 0.01 & 27 & 1 \\
\hline 36 & $24 / 05 / 197821: 00$ & 15 & 0.36 & 1.69 & 1.34 & 0.05 & 4 & 1 \\
\hline 37 & 27/07/1903 17:00 & 15 & 0.36 & 1.60 & 1.24 & 0.03 & 16 & 1 \\
\hline 38 & $7 / 06 / 200820: 00$ & 14 & 0.36 & 1.10 & 0.74 & -0.02 & 11 & 2 \\
\hline 39 & 25/04/1990 07:00 & 14 & 0.38 & 1.26 & 0.88 & 0.16 & 8 & 1 \\
\hline 40 & 19/08/2001 16:00 & 14 & 0.26 & 1.60 & 1.34 & 0.01 & 16 & 2 \\
\hline 41 & 11/07/1926 20:00 & 12 & 0.75 & 2.08 & 1.32 & 0.09 & 5 & 1 \\
\hline 42 & 3/01/2006 03:00 & 12 & 0.90 & 1.99 & 1.10 & 0.16 & 18 & 4 \\
\hline 43 & 14/07/1987 23:00 & 12 & 0.39 & 1.66 & 1.27 & 0.02 & 4 & 1 \\
\hline 44 & 9/07/1918 16:00 & 12 & 0.48 & 1.61 & 1.13 & 0.13 & 16 & 2 \\
\hline 45 & 7/03/1954 09:00 & 12 & 0.38 & 2.07 & 1.70 & 0.10 & 5 & 1 \\
\hline 46 & 24/05/2002 02:00 & 12 & 0.46 & 1.07 & 0.61 & 0.07 & 11 & 1 \\
\hline 47 & $30 / 03 / 2002$ 17:00 & 12 & 0.30 & 1.58 & 1.29 & 0.05 & 16 & 3 \\
\hline 48 & 21/06/1974 16:00 & 11 & 0.42 & 1.58 & 1.16 & 0.12 & 16 & 1 \\
\hline 49 & 26/09/1984 23:00 & 11 & 0.24 & 2.21 & 1.97 & 0.03 & 24 & 1 \\
\hline 50 & 14/07/1956 00:00 & 11 & 0.46 & 2.07 & 1.61 & 0.14 & 5 & 1 \\
\hline 51 & 27/07/1938 19:00 & 10 & 0.33 & 2.04 & 1.71 & 0.16 & 5 & 2 \\
\hline 52 & $2 / 08 / 199622: 00$ & 10 & 0.24 & 1.25 & 1.01 & 0.08 & 8 & 1 \\
\hline
\end{tabular}




\begin{tabular}{|c|c|c|c|c|c|c|c|c|}
\hline 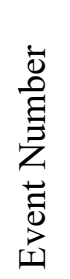 & 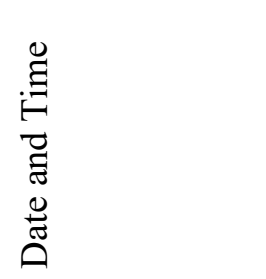 & 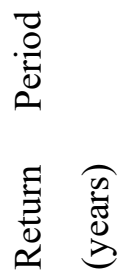 & 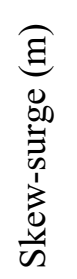 & 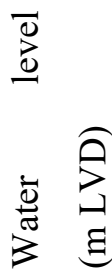 & 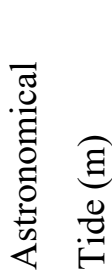 & $\begin{array}{l}\underset{\Xi}{\Xi} \\
\underset{\Sigma}{\Omega}\end{array}$ & 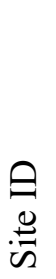 & 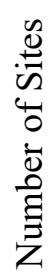 \\
\hline 53 & 18/04/1976 23:00 & 10 & 0.39 & 1.65 & 1.26 & 0.16 & 4 & 1 \\
\hline 54 & 20/06/1947 21:00 & 10 & 0.39 & 2.06 & 1.67 & 0.09 & 5 & 2 \\
\hline 55 & 21/10/1957 13:00 & 10 & 0.43 & 1.57 & 1.14 & 0.05 & 16 & 1 \\
\hline 56 & 23/02/1925 02:00 & 9 & 0.45 & 1.57 & 1.12 & 0.17 & 16 & 1 \\
\hline 57 & 15/03/1903 16:00 & 9 & 0.46 & 1.57 & 1.11 & 0.13 & 16 & 1 \\
\hline 58 & 16/05/1972 19:00 & 9 & 0.26 & 1.06 & 0.79 & 0.10 & 11 & 1 \\
\hline 59 & 19/09/1993 22:00 & 9 & 0.31 & 1.64 & 1.33 & -0.06 & 4 & 1 \\
\hline 60 & 18/06/1924 16:00 & 8 & 0.35 & 1.56 & 1.21 & 0.08 & 16 & 1 \\
\hline 61 & 1/03/2010 14:00 & 8 & 0.33 & 1.66 & 1.33 & 0.04 & 19 & 1 \\
\hline 62 & 22/05/2018 23:00 & 8 & 0.33 & 1.05 & 0.72 & 0.03 & 11 & 1 \\
\hline 63 & $14 / 05 / 1956$ 19:00 & 7 & 0.42 & 1.61 & 1.19 & 0.04 & 14 & 1 \\
\hline 64 & 27/05/1903 15:00 & 7 & 0.33 & 1.55 & 1.22 & 0.13 & 16 & 1 \\
\hline 65 & 5/06/2012 23:00 & 7 & 0.59 & 2.08 & 1.49 & 0.20 & 21 & 1 \\
\hline 66 & $8 / 08 / 195222: 00$ & 6 & 0.34 & 2.01 & 1.67 & -0.03 & 5 & 1 \\
\hline 67 & 12/01/2001 09:00 & 6 & 0.21 & 1.53 & 1.31 & 0.02 & 3 & 2 \\
\hline 68 & 24/09/2013 22:00 & 6 & 0.53 & 1.22 & 0.70 & 0.02 & 6 & 1 \\
\hline 69 & 3/06/2000 23:00 & 6 & 0.40 & 2.17 & 1.77 & 0.13 & 24 & 3 \\
\hline 70 & 26/12/2003 06:00 & 6 & 0.31 & 1.55 & 1.23 & 0.06 & 16 & 1 \\
\hline 71 & 13/12/2008 06:00 & 6 & 0.39 & 1.19 & 0.80 & 0.09 & 10 & 1 \\
\hline 72 & 21/04/1936 15:00 & 6 & 0.39 & 1.60 & 1.22 & 0.02 & 14 & 1 \\
\hline 73 & 31/12/1955 09:00 & 6 & 0.22 & 2.00 & 1.78 & 0.10 & 5 & 1 \\
\hline 74 & $30 / 06 / 193821: 00$ & 6 & 0.15 & 2.00 & 1.84 & 0.14 & 5 & 1 \\
\hline 75 & 9/06/1918 15:00 & 6 & 0.37 & 1.54 & 1.17 & 0.19 & 16 & 1 \\
\hline 76 & 9/10/1971 07:00 & 6 & 0.33 & 1.04 & 0.71 & 0.08 & 11 & 1 \\
\hline 77 & $30 / 07 / 200822: 00$ & 6 & 0.80 & 2.45 & 1.65 & 0.13 & 29 & 2 \\
\hline 78 & 11/07/1971 22:00 & 6 & 0.45 & 1.60 & 1.16 & 0.23 & 4 & 1 \\
\hline 79 & 6/05/2013 07:00 & 6 & 1.15 & 2.23 & 1.08 & 0.23 & 25 & 1 \\
\hline 80 & $30 / 04 / 201720: 00$ & 5 & 0.28 & 1.04 & 0.76 & 0.01 & 11 & 1 \\
\hline
\end{tabular}




\begin{tabular}{|c|c|c|c|c|c|c|c|c|}
\hline 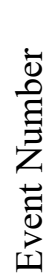 & 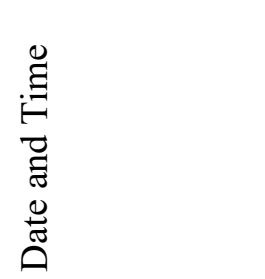 & 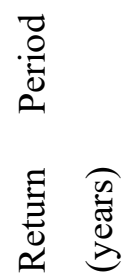 & 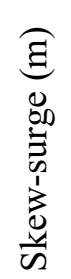 & 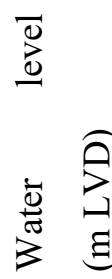 & 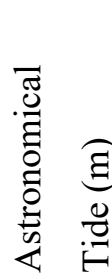 & 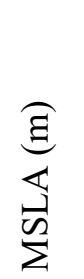 & $\begin{array}{l}\ominus \\
\stackrel{0}{*}\end{array}$ & 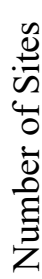 \\
\hline 81 & $13 / 04 / 2001$ 10:00 & 5 & 0.53 & 1.30 & 0.77 & 0.06 & 9 & 1 \\
\hline 82 & 23/08/1989 00:00 & 5 & 0.45 & 1.60 & 1.15 & 0.17 & 4 & 1 \\
\hline 83 & 4/12/1951 08:00 & 5 & 0.29 & 1.03 & 0.75 & 0.08 & 11 & 1 \\
\hline 84 & 17/11/2016 09:00 & 5 & 0.21 & 2.13 & 1.92 & -0.01 & 7 & 1 \\
\hline 85 & $11 / 05 / 201222: 00$ & 5 & 0.33 & 1.18 & 0.85 & 0.11 & 10 & 1 \\
\hline
\end{tabular}


Table S3. Extreme skew-surge events.

\begin{tabular}{|c|c|c|c|c|c|c|c|}
\hline 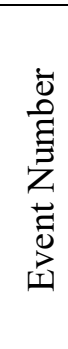 & 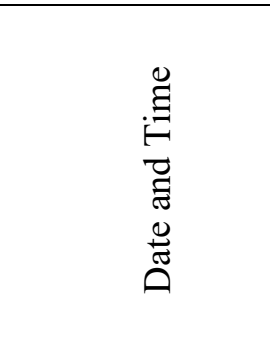 & 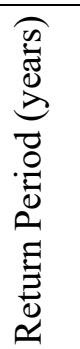 & 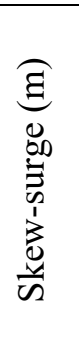 & 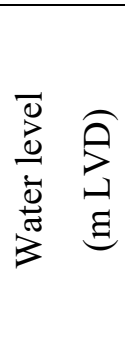 & 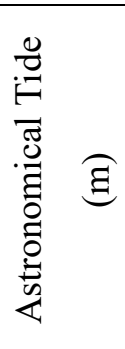 & 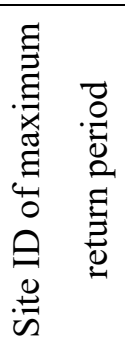 & 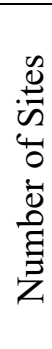 \\
\hline 1 & $25 / 12 / 199011: 00$ & 328 & 0.58 & 0.94 & 0.35 & 11 & 2 \\
\hline 2 & $22 / 01 / 201704: 00$ & 257 & 0.83 & 1.57 & 0.74 & 22 & 3 \\
\hline 3 & 1/12/2008 08:00 & 247 & 0.43 & 1.03 & 0.60 & 10 & 1 \\
\hline 4 & 2/04/1975 08:00 & 224 & 0.59 & 1.31 & 0.72 & 16 & 1 \\
\hline 5 & $6 / 05 / 201307: 00$ & 182 & 1.15 & 2.23 & 1.08 & 25 & 8 \\
\hline 6 & $12 / 06 / 2006$ 10:00 & 173 & 0.54 & 1.25 & 0.71 & 23 & 4 \\
\hline 7 & $26 / 07 / 200814: 00$ & 159 & 0.69 & 1.47 & 0.79 & 1 & 7 \\
\hline 8 & $11 / 07 / 192620: 00$ & 146 & 0.75 & 2.08 & 1.32 & 5 & 1 \\
\hline 9 & $30 / 05 / 195607: 00$ & 142 & 0.64 & 1.22 & 0.58 & 14 & 3 \\
\hline 10 & $6 / 07 / 1939$ 18:00 & 128 & 0.64 & 1.40 & 0.76 & 14 & 2 \\
\hline 11 & $17 / 04 / 199917: 00$ & 101 & 0.55 & 1.69 & 1.13 & 13 & 3 \\
\hline 12 & 17/05/1994 06:00 & 84 & 1.12 & 1.87 & 0.75 & 18 & 1 \\
\hline 13 & $12 / 07 / 2009$ 11:00 & 76 & 0.50 & 1.18 & 0.68 & 2 & 3 \\
\hline 14 & 20/06/1918 11:00 & 67 & 0.56 & 1.32 & 0.76 & 16 & 1 \\
\hline 15 & $1 / 02 / 201811: 00$ & 65 & 0.84 & 2.49 & 1.65 & 21 & 5 \\
\hline 16 & 1/03/1951 01:00 & 63 & 0.68 & 1.91 & 1.23 & 5 & 1 \\
\hline 17 & $16 / 04 / 2003$ 18:00 & 62 & 0.59 & 1.43 & 0.84 & 9 & 1 \\
\hline 18 & 21/08/2003 02:00 & 60 & 0.56 & 1.04 & 0.48 & 6 & 2 \\
\hline 19 & $16 / 03 / 1980$ 15:00 & 57 & 0.56 & 1.70 & 1.14 & 16 & 1 \\
\hline 20 & 4/03/2003 02:00 & 54 & 0.57 & 1.55 & 0.98 & 19 & 1 \\
\hline 21 & $5 / 01 / 201810: 00$ & 51 & 0.62 & 2.50 & 1.87 & 7 & 3 \\
\hline 22 & $21 / 05 / 201622: 00$ & 47 & 0.71 & 1.98 & 1.27 & 30 & 3 \\
\hline 23 & 17/05/1924 17:00 & 47 & 0.66 & 1.93 & 1.27 & 5 & 1 \\
\hline 24 & 3/03/1965 03:00 & 45 & 0.60 & 1.39 & 0.78 & 14 & 1 \\
\hline
\end{tabular}




\begin{tabular}{|c|c|c|c|c|c|c|c|}
\hline 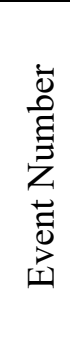 & 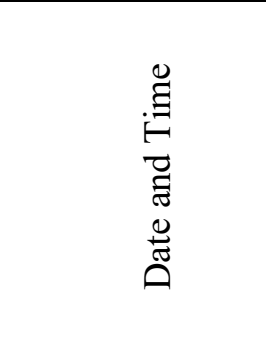 & 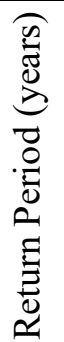 & 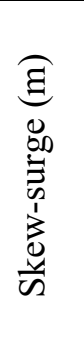 & 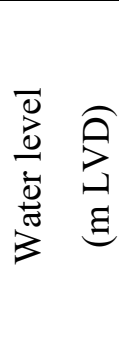 & 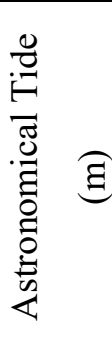 & 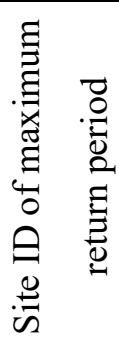 & 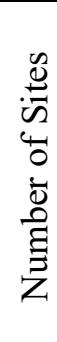 \\
\hline 25 & $3 / 06 / 195502: 00$ & 45 & 0.60 & 1.30 & 0.70 & 14 & 1 \\
\hline 26 & 21/07/2017 13:00 & 44 & 0.57 & 1.63 & 1.06 & 15 & 4 \\
\hline 27 & 13/06/1968 22:00 & 43 & 0.62 & 1.77 & 1.16 & 4 & 1 \\
\hline 28 & 24/09/2013 23:00 & 42 & 0.64 & 1.89 & 1.25 & 5 & 2 \\
\hline 29 & 28/05/1909 10:00 & 41 & 0.54 & 1.27 & 0.73 & 16 & 1 \\
\hline 30 & 17/04/2014 09:00 & 36 & 0.63 & 1.92 & 1.29 & 5 & 2 \\
\hline 31 & 8/09/1989 12:00 & 35 & 0.47 & 1.01 & 0.54 & 8 & 3 \\
\hline 32 & 15/04/1962 13:00 & 35 & 0.50 & 0.95 & 0.45 & 11 & 2 \\
\hline 33 & $23 / 11 / 198916: 00$ & 33 & 0.47 & 1.05 & 0.58 & 8 & 1 \\
\hline 34 & $13 / 04 / 2001 \quad 10: 00$ & 33 & 0.53 & 1.30 & 0.77 & 9 & 1 \\
\hline 35 & 21/06/2013 01:00 & 32 & 0.50 & 1.11 & 0.61 & 11 & 1 \\
\hline 36 & 17/05/2016 02:00 & 32 & 0.50 & 0.98 & 0.48 & 11 & 1 \\
\hline 37 & $12 / 05 / 201620: 00$ & 31 & 0.50 & 1.31 & 0.81 & 17 & 2 \\
\hline 38 & $14 / 03 / 2007$ 19:00 & 24 & 0.64 & 1.43 & 0.79 & 27 & 2 \\
\hline 39 & $15 / 10 / 1918$ 10:00 & 23 & 0.52 & 1.38 & 0.86 & 16 & 1 \\
\hline 40 & 1/05/1936 11:00 & 20 & 0.52 & 1.23 & 0.71 & 16 & 2 \\
\hline 41 & 30/12/1996 23:00 & 19 & 0.45 & 1.07 & 0.62 & 8 & 1 \\
\hline 42 & 26/03/1936 09:00 & 19 & 0.59 & 2.24 & 1.66 & 5 & 1 \\
\hline 43 & $6 / 07 / 2011 \quad 19: 00$ & 19 & 0.49 & 1.34 & 0.85 & 17 & 1 \\
\hline 44 & 11/04/1968 02:00 & 19 & 0.57 & 1.45 & 0.88 & 14 & 1 \\
\hline 45 & 18/01/1980 16:00 & 18 & 0.57 & 1.41 & 0.84 & 14 & 1 \\
\hline 46 & $16 / 05 / 196020: 00$ & 17 & 0.51 & 1.50 & 0.99 & 16 & 1 \\
\hline 47 & 7/06/2010 05:00 & 17 & 0.50 & 1.35 & 0.85 & 24 & 1 \\
\hline 48 & $30 / 05 / 1972$ 17:00 & 17 & 0.57 & 1.29 & 0.72 & 14 & 1 \\
\hline 49 & 1/01/1958 12:00 & 17 & 0.56 & 1.20 & 0.63 & 14 & 1 \\
\hline
\end{tabular}




\begin{tabular}{|c|c|c|c|c|c|c|c|}
\hline 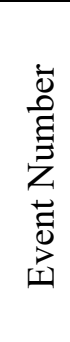 & 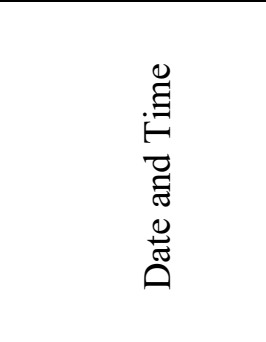 & 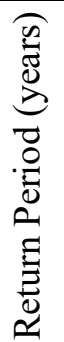 & 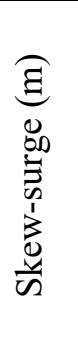 & 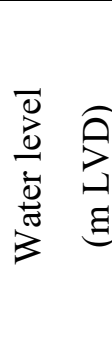 & 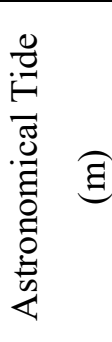 & 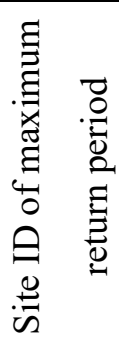 & 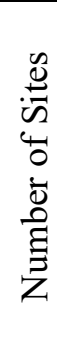 \\
\hline 50 & 14/06/1975 23:00 & 17 & 0.48 & 1.39 & 0.91 & 9 & 1 \\
\hline 51 & 14/08/1954 16:00 & 16 & 0.47 & 1.00 & 0.52 & 11 & 1 \\
\hline 52 & 29/11/1998 15:00 & 16 & 0.43 & 1.27 & 0.84 & 3 & 2 \\
\hline 53 & 18/09/2005 06:00 & 16 & 0.55 & 2.03 & 1.47 & 7 & 1 \\
\hline 54 & 26/01/1986 16:00 & 14 & 0.56 & 1.25 & 0.69 & 14 & 1 \\
\hline 55 & $3 / 12 / 2004$ 18:00 & 14 & 0.99 & 1.46 & 0.47 & 18 & 1 \\
\hline 56 & 15/06/1974 15:00 & 14 & 0.47 & 0.99 & 0.53 & 9 & 1 \\
\hline 57 & 9/07/1961 13:00 & 14 & 0.50 & 1.33 & 0.83 & 16 & 1 \\
\hline 58 & 24/02/2008 01:00 & 13 & 0.60 & 1.52 & 0.92 & 20 & 1 \\
\hline 59 & 13/04/1981 02:00 & 13 & 0.55 & 1.78 & 1.23 & 5 & 1 \\
\hline 60 & $\begin{array}{l}5 / 05 / 200105: 00 \\
\end{array}$ & 13 & 0.42 & 1.41 & 0.98 & 3 & 1 \\
\hline 61 & 7/07/1978 16:00 & 11 & 0.50 & 1.27 & 0.77 & 16 & 1 \\
\hline 62 & $23 / 01 / 2011 \quad 10: 00$ & 11 & 0.42 & 1.71 & 1.29 & 3 & 2 \\
\hline 63 & 13/08/1918 19:00 & 11 & 0.50 & 1.14 & 0.64 & 16 & 1 \\
\hline 64 & $24 / 05 / 200202: 00$ & 11 & 0.46 & 1.07 & 0.61 & 11 & 1 \\
\hline 65 & 24/03/1964 13:00 & 11 & 0.49 & 1.25 & 0.75 & 16 & 1 \\
\hline 66 & 12/03/1975 07:00 & 10 & 0.54 & 1.63 & 1.09 & 5 & 2 \\
\hline 67 & 19/07/1978 06:00 & 10 & 0.52 & 1.60 & 1.08 & 4 & 2 \\
\hline 68 & 18/03/2002 08:00 & 10 & 0.39 & 0.89 & 0.51 & 12 & 2 \\
\hline 69 & 11/06/2014 05:00 & 10 & 0.44 & 1.11 & 0.67 & 9 & 1 \\
\hline 70 & 25/04/2009 23:00 & 10 & 0.59 & 1.60 & 1.01 & 20 & 2 \\
\hline 71 & $6 / 05 / 200907: 00$ & 10 & 0.52 & 1.65 & 1.13 & 28 & 1 \\
\hline 72 & 25/05/2015 23:00 & 10 & 0.54 & 1.30 & 0.75 & 14 & 1 \\
\hline 73 & $3 / 05 / 1934$ 10:00 & 10 & 0.53 & 1.86 & 1.32 & 5 & 1 \\
\hline 74 & 1/07/1997 04:00 & 10 & 0.44 & 1.21 & 0.77 & 9 & 1 \\
\hline
\end{tabular}




\begin{tabular}{|c|c|c|c|c|c|c|c|}
\hline 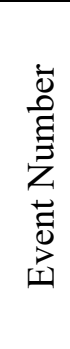 & 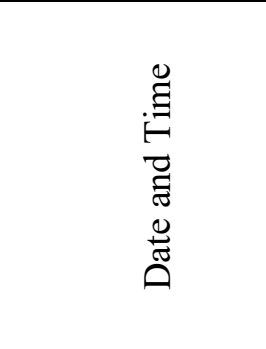 & 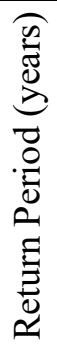 & 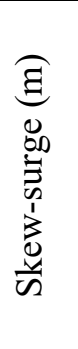 & 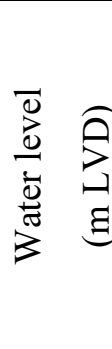 & 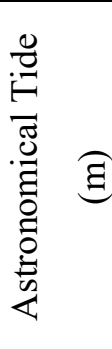 & 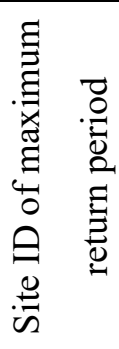 & 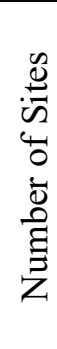 \\
\hline 75 & 11/03/1997 22:00 & 10 & 0.52 & 1.81 & 1.29 & 4 & 1 \\
\hline 76 & 11/06/1971 04:00 & 10 & 0.49 & 1.17 & 0.68 & 16 & 1 \\
\hline 77 & 4/04/2001 13:00 & 10 & 0.50 & 1.34 & 0.84 & 13 & 1 \\
\hline 78 & 24/04/1990 18:00 & 10 & 0.42 & 1.17 & 0.76 & 8 & 1 \\
\hline 79 & 6/10/2005 06:00 & 9 & 0.50 & 1.33 & 0.83 & 13 & 1 \\
\hline 80 & 12/12/1996 23:00 & 9 & 0.48 & 2.00 & 1.52 & 24 & 1 \\
\hline 81 & 18/08/2000 12:00 & 9 & 0.48 & 1.73 & 1.25 & 24 & 1 \\
\hline 82 & 2/07/1998 11:00 & 9 & 0.45 & 0.88 & 0.43 & 11 & 1 \\
\hline 83 & 12/04/1936 19:00 & 9 & 0.54 & 1.28 & 0.75 & 14 & 1 \\
\hline 84 & 11/07/2007 17:00 & 9 & 0.51 & 1.44 & 0.93 & 4 & 1 \\
\hline 85 & 16/04/1910 09:00 & 8 & 0.48 & 1.28 & 0.80 & 16 & 1 \\
\hline 86 & $30 / 07 / 200822: 00$ & 8 & 0.80 & 2.45 & 1.65 & 29 & 1 \\
\hline 87 & 8/07/2013 01:00 & 8 & 0.94 & 1.61 & 0.67 & 18 & 1 \\
\hline 88 & $9 / 07 / 1918$ 16:00 & 8 & 0.48 & 1.61 & 1.13 & 16 & 1 \\
\hline 89 & $3 / 10 / 1971$ 14:00 & 8 & 0.48 & 1.47 & 0.99 & 16 & 1 \\
\hline 90 & 17/04/1972 19:00 & 8 & 0.44 & 1.20 & 0.75 & 11 & 1 \\
\hline 91 & 14/12/1999 09:00 & 8 & 0.49 & 1.20 & 0.71 & 13 & 1 \\
\hline 92 & 20/09/1971 08:00 & 8 & 0.51 & 1.66 & 1.16 & 5 & 1 \\
\hline 93 & 11/03/2016 03:00 & 7 & 0.93 & 2.11 & 1.18 & 18 & 1 \\
\hline 94 & 18/01/2011 21:00 & 7 & 0.54 & 1.81 & 1.27 & 22 & 2 \\
\hline 95 & 16/05/1951 11:00 & 7 & 0.53 & 1.24 & 0.71 & 14 & 1 \\
\hline 96 & 20/07/2014 02:00 & 7 & 0.50 & 1.50 & 1.00 & 4 & 1 \\
\hline 97 & 17/02/2008 01:00 & 7 & 0.49 & 1.42 & 0.93 & 13 & 1 \\
\hline 98 & $21 / 02 / 2004$ 18:00 & 7 & 0.37 & 1.02 & 0.66 & 12 & 1 \\
\hline 99 & $30 / 07 / 201204: 00$ & 7 & 0.40 & 1.06 & 0.66 & 8 & 1 \\
\hline
\end{tabular}




\begin{tabular}{|c|c|c|c|c|c|c|c|}
\hline 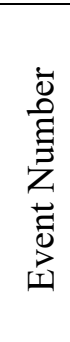 & 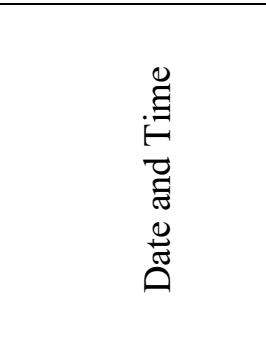 & 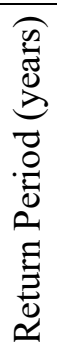 & 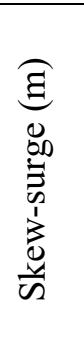 & 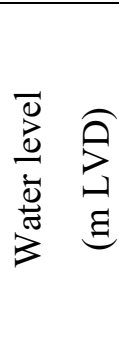 & 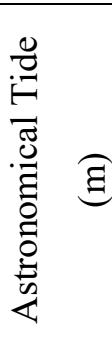 & 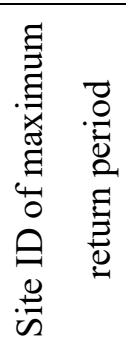 & 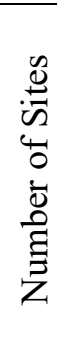 \\
\hline 100 & $24 / 06 / 1903$ 01:00 & 7 & 0.48 & 1.53 & 1.05 & 16 & 1 \\
\hline 101 & $22 / 06 / 2007$ 10:00 & 7 & 0.47 & 1.16 & 0.68 & 16 & 3 \\
\hline 102 & 18/06/1978 04:00 & 7 & 0.50 & 1.63 & 1.13 & 5 & 1 \\
\hline 103 & 6/07/1903 13:00 & 7 & 0.47 & 1.34 & 0.87 & 16 & 1 \\
\hline 104 & 10/06/2016 19:00 & 7 & 0.46 & 1.20 & 0.74 & 17 & 2 \\
\hline 105 & 13/12/2008 06:00 & 7 & 0.39 & 1.19 & 0.80 & 10 & 1 \\
\hline 106 & 16/03/2015 03:00 & 7 & 0.49 & 1.34 & 0.84 & 4 & 1 \\
\hline 107 & 29/07/1990 13:00 & 7 & 0.40 & 1.03 & 0.64 & 3 & 1 \\
\hline 108 & 8/07/2018 18:00 & 7 & 0.47 & 1.36 & 0.89 & 24 & 1 \\
\hline 109 & $10 / 05 / 193218: 00$ & 7 & 0.52 & 1.33 & 0.81 & 14 & 1 \\
\hline 110 & $14 / 02 / 191822: 00$ & 7 & 0.50 & 2.11 & 1.61 & 5 & 1 \\
\hline 111 & 15/06/1909 00:00 & 7 & 0.47 & 1.29 & 0.81 & 16 & 1 \\
\hline 112 & 16/02/2004 03:00 & 7 & 0.50 & 1.62 & 1.12 & 7 & 1 \\
\hline 113 & $26 / 07 / 1939$ 12:00 & 6 & 0.52 & 1.49 & 0.97 & 14 & 1 \\
\hline 114 & 15/03/2014 19:00 & 6 & 0.49 & 1.50 & 1.00 & 5 & 1 \\
\hline 115 & 6/05/1971 12:00 & 6 & 0.47 & 1.13 & 0.66 & 16 & 1 \\
\hline 116 & $28 / 04 / 1938$ 17:00 & 6 & 0.49 & 1.60 & 1.11 & 5 & 1 \\
\hline 117 & 14/01/1949 07:00 & 6 & 0.49 & 1.65 & 1.16 & 5 & 1 \\
\hline 118 & 15/06/1999 18:00 & 6 & 0.36 & 1.41 & 1.05 & 12 & 1 \\
\hline 119 & 5/06/1980 08:00 & 6 & 0.47 & 1.29 & 0.82 & 16 & 1 \\
\hline 120 & 28/12/2010 03:00 & 6 & 0.53 & 1.91 & 1.39 & 22 & 1 \\
\hline 121 & $22 / 05 / 1980$ 10:00 & 6 & 0.47 & 1.18 & 0.71 & 16 & 1 \\
\hline 122 & 28/04/1932 13:00 & 6 & 0.49 & 1.48 & 0.99 & 5 & 1 \\
\hline 123 & $6 / 03 / 2015$ 11:00 & 6 & 0.52 & 2.16 & 1.64 & 22 & 1 \\
\hline 124 & 25/05/1992 01:00 & 6 & 0.39 & 0.97 & 0.58 & 8 & 1 \\
\hline
\end{tabular}




\begin{tabular}{|c|c|c|c|c|c|c|c|}
\hline 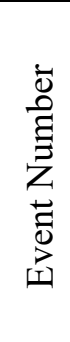 & 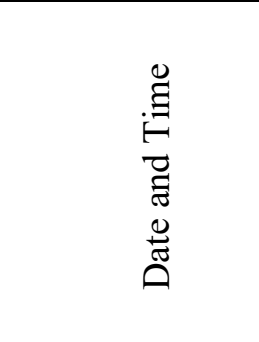 & 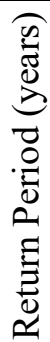 & 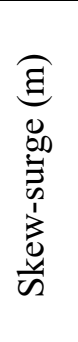 & 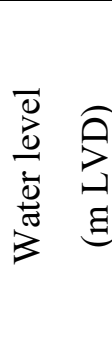 & 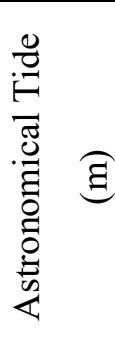 & 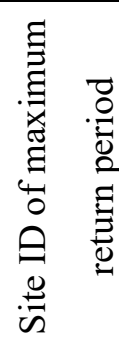 & 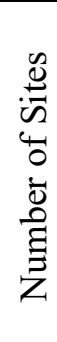 \\
\hline 125 & $18 / 06 / 201222: 00$ & 6 & 0.61 & 1.63 & 1.02 & 21 & 1 \\
\hline 126 & 14/07/1918 19:00 & 6 & 0.47 & 1.20 & 0.73 & 16 & 1 \\
\hline 127 & 9/04/1966 19:00 & 5 & 0.46 & 1.31 & 0.84 & 16 & 1 \\
\hline 128 & 8/04/1969 20:00 & 5 & 0.52 & 1.50 & 0.98 & 14 & 1 \\
\hline 129 & $22 / 09 / 2014$ 10:00 & 5 & 0.57 & 1.84 & 1.27 & 27 & 1 \\
\hline 130 & 11/05/1916 01:00 & 5 & 0.48 & 1.40 & 0.92 & 5 & 1 \\
\hline 131 & $24 / 01 / 200615: 00$ & 5 & 0.48 & 1.38 & 0.90 & 5 & 1 \\
\hline 132 & $7 / 06 / 2008$ 16:00 & 5 & 0.91 & 1.89 & 0.98 & 18 & 1 \\
\hline 133 & 11/12/2001 21:00 & 5 & 0.55 & 1.41 & 0.86 & 20 & 1 \\
\hline 134 & 9/04/1982 20:00 & 5 & 0.47 & 1.48 & 1.01 & 4 & 1 \\
\hline 135 & 13/12/2002 02:00 & 5 & 0.49 & 1.40 & 0.91 & 7 & 1 \\
\hline
\end{tabular}


Table S4. Largest recorded return level sea levels at each site.

\begin{tabular}{|c|c|c|c|c|c|c|}
\hline 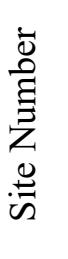 & 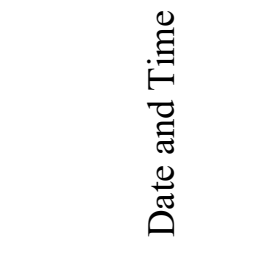 & 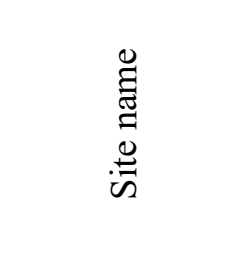 & 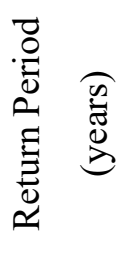 & 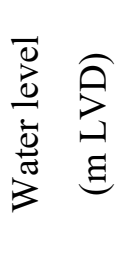 & 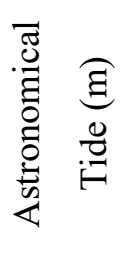 & 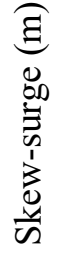 \\
\hline 1 & $23 / 01 / 201111: 00$ & Rangaunu & 16 & 1.69 & 1.36 & 0.33 \\
\hline 2 & $23 / 01 / 201110: 00$ & Whangaroa & 48 & 1.60 & 1.27 & 0.33 \\
\hline 3 & 23/01/2011 10:00 & Opua & 330 & 1.71 & 1.29 & 0.42 \\
\hline 4 & 11/03/1997 22:00 & Marsden Point & 84 & 1.81 & 1.29 & 0.52 \\
\hline 5 & 26/03/1936 09:00 & Auckland & 131 & 2.24 & 1.66 & 0.59 \\
\hline 6 & $23 / 01 / 201110: 00$ & Whitianga & 16 & 1.30 & 0.95 & 0.36 \\
\hline 7 & $5 / 01 / 2018$ 10:00 & Tararu & 770 & 2.50 & 1.87 & 0.62 \\
\hline 8 & $30 / 08 / 200020: 00$ & Tauranga & 32 & 1.28 & 1.01 & 0.28 \\
\hline 9 & 5/01/2018 09:00 & Moturiki & 62 & 1.45 & 1.12 & 0.33 \\
\hline 10 & 28/05/2013 21:00 & Gisborne & 10 & 1.21 & 0.90 & 0.31 \\
\hline 11 & 17/04/1972 19:00 & Wellington & 87 & 1.20 & 0.75 & 0.44 \\
\hline 12 & 15/06/1999 18:00 & Kaikoura & 942 & 1.41 & 1.05 & 0.36 \\
\hline 13 & 17/04/1999 17:00 & Sumner & 65 & 1.69 & 1.13 & 0.55 \\
\hline 14 & $2 / 02 / 201805: 00$ & Lyttelton & 114 & 1.79 & 1.26 & 0.53 \\
\hline 15 & 21/07/2017 13:00 & Timaru & 67 & 1.63 & 1.06 & 0.57 \\
\hline 16 & 4/08/1921 15:00 & Dunedin & 69 & 1.70 & 1.25 & 0.45 \\
\hline 17 & 26/05/2013 16:00 & Green Island & 25 & 1.44 & 1.12 & 0.32 \\
\hline 18 & 30/03/1998 03:00 & Invercargill & 65 & 2.17 & 1.28 & 0.89 \\
\hline 19 & 26/05/2013 14:00 & Dog Island & 50 & 1.72 & 1.35 & 0.37 \\
\hline 20 & $1 / 02 / 201812: 00$ & Jackson Bay & $>1000$ & 2.01 & 1.32 & 0.69 \\
\hline 21 & $1 / 02 / 201811: 00$ & Charleston & $>1000$ & 2.49 & 1.65 & 0.84 \\
\hline 22 & $1 / 02 / 201810: 00$ & Nelson & 33 & 2.61 & 2.06 & 0.55 \\
\hline 23 & $23 / 07 / 200923: 00$ & Kapiti & 62 & 1.36 & 0.97 & 0.40 \\
\hline 24 & $1 / 02 / 201810: 00$ & Taranaki & 101 & 2.33 & 1.85 & 0.48 \\
\hline 25 & $5 / 01 / 201813: 00$ & Kawhia & 31 & 2.39 & 1.79 & 0.60 \\
\hline 26 & $5 / 01 / 201813: 00$ & Raglan & 13 & 2.39 & 1.70 & 0.69 \\
\hline
\end{tabular}




\begin{tabular}{|c|l|l|c|c|c|c|}
\hline 27 & $5 / 01 / 201813: 00$ & Onehunga & 17 & 2.37 & 1.95 & 0.42 \\
\hline 28 & $17 / 04 / 199910: 00$ & Anawhata & 249 & 2.16 & 1.58 & 0.58 \\
\hline 29 & $19 / 09 / 200500: 00$ & Helensville & 14 & 2.56 & 2.10 & 0.46 \\
\hline 30 & $18 / 09 / 200523: 00$ & Pouto Point & 19 & 2.23 & 1.80 & 0.43 \\
\hline
\end{tabular}


Table S5. Largest recorded skew-surges at each site.

\begin{tabular}{|c|c|c|c|c|c|c|}
\hline 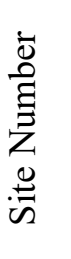 & 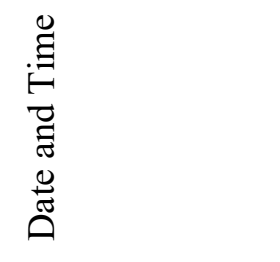 & 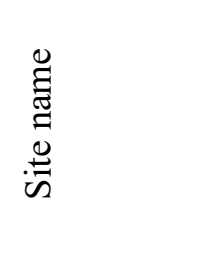 & 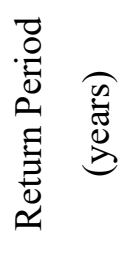 & 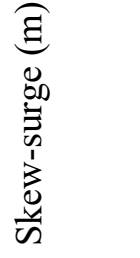 & 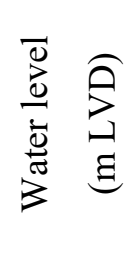 & 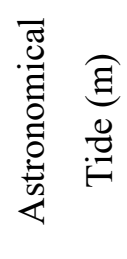 \\
\hline 1 & $26 / 07 / 200814: 00$ & Rangaunu & 159 & 0.69 & 1.47 & 0.79 \\
\hline 2 & 12/07/2009 11:00 & Whangaroa & 76 & 0.50 & 1.18 & 0.68 \\
\hline 3 & 26/07/2008 14:00 & Opua & 18 & 0.44 & 1.20 & 0.77 \\
\hline 4 & 12/07/2009 11:00 & Marsden Point & 46 & 0.63 & 1.35 & 0.72 \\
\hline 5 & 11/07/1926 20:00 & Auckland & 146 & 0.75 & 2.08 & 1.32 \\
\hline 6 & 21/08/2003 02:00 & Whitianga & 60 & 0.56 & 1.04 & 0.48 \\
\hline 7 & $5 / 01 / 201810: 00$ & Tararu & 51 & 0.62 & 2.50 & 1.87 \\
\hline 8 & 8/09/1989 12:00 & Tauranga & 35 & 0.47 & 1.01 & 0.54 \\
\hline 9 & 16/04/2003 18:00 & Moturiki & 62 & 0.59 & 1.43 & 0.84 \\
\hline 10 & 1/12/2008 08:00 & Gisborne & 247 & 0.43 & 1.03 & 0.60 \\
\hline 11 & 25/12/1990 11:00 & Wellington & 328 & 0.58 & 0.94 & 0.35 \\
\hline 12 & 18/03/2002 08:00 & Kaikoura & 10 & 0.39 & 0.89 & 0.51 \\
\hline 13 & 17/04/1999 17:00 & Sumner & 101 & 0.55 & 1.69 & 1.13 \\
\hline 14 & $30 / 05 / 1956$ 07:00 & Lyttelton & 142 & 0.64 & 1.22 & 0.58 \\
\hline 15 & 21/07/2017 13:00 & Timaru & 44 & 0.57 & 1.63 & 1.06 \\
\hline 16 & 2/04/1975 08:00 & Dunedin & 224 & 0.59 & 1.31 & 0.72 \\
\hline 17 & $12 / 05 / 201620: 00$ & Green Island & 31 & 0.50 & 1.31 & 0.81 \\
\hline 18 & 17/05/1994 06:00 & Invercargill & 84 & 1.12 & 1.87 & 0.75 \\
\hline 19 & 4/03/2003 02:00 & Dog Island & 54 & 0.57 & 1.55 & 0.98 \\
\hline 20 & $1 / 02 / 201812: 00$ & Jackson Bay & 63 & 0.69 & 2.01 & 1.32 \\
\hline 21 & $1 / 02 / 201811: 00$ & Charleston & 65 & 0.84 & 2.49 & 1.65 \\
\hline 22 & 22/01/2017 04:00 & Nelson & 257 & 0.83 & 1.57 & 0.74 \\
\hline 23 & 12/06/2006 10:00 & Kapiti & 173 & 0.54 & 1.25 & 0.71 \\
\hline 24 & 6/05/2013 07:00 & Taranaki & 84 & 0.54 & 1.61 & 1.08 \\
\hline 25 & 6/05/2013 07:00 & Kawhia & 182 & 1.15 & 2.23 & 1.08 \\
\hline
\end{tabular}




\begin{tabular}{|c|l|l|c|c|c|c|}
\hline 26 & $6 / 05 / 201307: 00$ & Raglan & 28 & 1.15 & 2.17 & 1.02 \\
\hline 27 & $6 / 05 / 201308: 00$ & Onehunga & 147 & 0.71 & 1.96 & 1.25 \\
\hline 28 & $17 / 04 / 199910: 00$ & Anawhata & 49 & 0.58 & 2.16 & 1.58 \\
\hline 29 & $6 / 05 / 201308: 00$ & Helensville & 95 & 1.04 & 2.34 & 1.30 \\
\hline 30 & $21 / 05 / 201622: 00$ & Pouto Point & 47 & 0.71 & 1.98 & 1.27 \\
\hline
\end{tabular}


Table S6. Tidal amplitudes. The three dominant tidal harmonic constituents: principal lunar semidiurnal $\mathbf{M}_{2}$, principal solar semidiurnal $85 \mathbf{S}_{2}$ and larger lunar elliptic semidiurnal $\mathbf{N}_{2}$ are included along with the solar annual and semi-annual $\mathbf{S}_{a}$ and $\mathbf{S}_{\text {sa. }}$ MHWS7 is the height equalled or exceeded by the highest $7 \%$ of all high tides and HAT is highest astronomical tide.

\begin{tabular}{|c|c|c|c|c|c|c|c|c|}
\hline \multicolumn{2}{|c|}{ Site details } & \multicolumn{5}{|c|}{ Amplitude (m) of tidal harmonic constituent } & \multicolumn{2}{|c|}{ Tidal amplitude (m) } \\
\hline Number & Name & $M_{2}$ & $S_{2}$ & $N_{2}$ & $S a$ & Ssa & MHWS7 & HAT \\
\hline 1 & Rangaunu & 0.83 & 0.14 & 0.15 & 0.06 & 0.01 & 1.16 & 1.35 \\
\hline 2 & Whangaroa & 0.81 & 0.15 & 0.16 & 0.06 & 0.01 & 1.09 & 1.29 \\
\hline 3 & Opua & 0.82 & 0.14 & 0.17 & 0.05 & 0.01 & 1.11 & 1.34 \\
\hline 4 & Marsden Point & 0.89 & 0.14 & 0.18 & 0.05 & 0.01 & 1.19 & 1.43 \\
\hline 5 & Auckland & 1.15 & 0.18 & 0.23 & 0.04 & 0.01 & 1.54 & 1.89 \\
\hline 6 & Whitianga & 0.66 & 0.08 & 0.14 & 0.05 & 0.01 & 0.86 & 1.05 \\
\hline 7 & Tararu & 1.26 & 0.19 & 0.26 & 0.04 & 0.00 & 1.65 & 1.93 \\
\hline 8 & Tauranga & 0.70 & 0.09 & 0.14 & 0.04 & 0.00 & 0.90 & 1.07 \\
\hline 9 & Moturiki & 0.73 & 0.10 & 0.16 & 0.04 & 0.01 & 0.96 & 1.14 \\
\hline 10 & Gisborne & 0.64 & 0.05 & 0.14 & 0.04 & 0.01 & 0.82 & 0.96 \\
\hline 11 & Wellington & 0.47 & 0.03 & 0.12 & 0.02 & 0.01 & 0.66 & 0.83 \\
\hline 12 & Kaikoura & 0.67 & 0.02 & 0.15 & 0.03 & 0.02 & 0.87 & 1.05 \\
\hline 13 & Sumner & 0.83 & 0.05 & 0.19 & 0.03 & 0.02 & 1.08 & 1.34 \\
\hline 14 & Lyttelton & 0.83 & 0.05 & 0.19 & 0.03 & 0.01 & 1.12 & 1.39 \\
\hline 15 & Timaru & 0.78 & 0.09 & 0.18 & 0.03 & 0.01 & 1.02 & 1.21 \\
\hline 16 & Dunedin & 0.75 & 0.08 & 0.17 & 0.02 & 0.01 & 1.08 & 1.37 \\
\hline 17 & Green Island & 0.73 & 0.10 & 0.17 & 0.02 & 0.01 & 0.98 & 1.17 \\
\hline 18 & Invercargill & 0.72 & 0.13 & 0.14 & 0.02 & 0.02 & 1.11 & 1.38 \\
\hline 19 & Dog Island & 0.89 & 0.18 & 0.21 & 0.01 & 0.01 & 1.21 & 1.44 \\
\hline 20 & Jackson Bay & 0.83 & 0.21 & 0.18 & 0.04 & 0.01 & 1.16 & 1.41 \\
\hline 21 & Charleston & 1.07 & 0.28 & 0.22 & 0.04 & 0.01 & 1.50 & 1.80 \\
\hline 22 & Nelson & 1.34 & 0.42 & 0.24 & 0.04 & 0.01 & 1.92 & 2.37 \\
\hline 23 & Kapiti & 0.55 & 0.26 & 0.08 & 0.02 & 0.01 & 0.90 & 1.10 \\
\hline 24 & Taranaki & 1.19 & 0.33 & 0.23 & 0.03 & 0.01 & 1.66 & 2.00 \\
\hline 25 & Kawhia & 1.16 & 0.31 & 0.22 & 0.05 & 0.01 & 1.63 & 1.94 \\
\hline 26 & Raglan & 1.12 & 0.30 & 0.21 & 0.05 & 0.01 & 1.55 & 1.83 \\
\hline 27 & Onehunga & 1.33 & 0.34 & 0.24 & 0.04 & 0.00 & 1.81 & 2.12 \\
\hline
\end{tabular}




\begin{tabular}{|l|l|l|l|l|l|l|l|l|}
\hline 28 & Anawhata & 1.05 & 0.29 & 0.20 & 0.05 & 0.01 & 1.46 & 1.74 \\
\hline 29 & Helensville & 1.37 & 0.33 & 0.23 & 0.05 & 0.00 & 1.89 & 2.22 \\
\hline 30 & Pouto Point & 1.14 & 0.28 & 0.21 & 0.04 & 0.01 & 1.56 & 1.84 \\
\hline
\end{tabular}


Table S7. Extreme sea-level return period height estimates (m) for each of the 30 study sites for different return periods. SSJPM = skewsurge joint-probability method, GPD = generalised Pareto distribution.

\begin{tabular}{|c|c|c|c|c|c|c|c|c|c|c|c|c|c|}
\hline \multirow{2}{*}{ 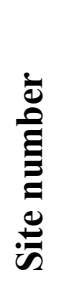 } & \multirow{2}{*}{ 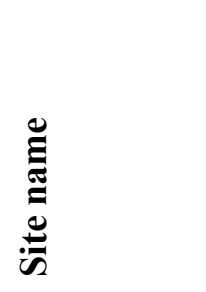 } & \multirow{2}{*}{ 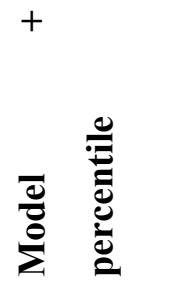 } & \multicolumn{11}{|c|}{ Return period (years) } \\
\hline & & & 1 & 2 & 5 & 10 & 25 & 50 & 75 & 100 & 250 & 500 & 1000 \\
\hline \multirow[t]{7}{*}{1} & \multirow[t]{7}{*}{ Rangaunu } & SSJPM $2.5 \%$ & 1.49 & 1.53 & 1.59 & 1.62 & 1.67 & 1.70 & 1.72 & 1.73 & 1.77 & 1.79 & 1.81 \\
\hline & & SSJPM 50\% & 1.50 & 1.55 & 1.61 & 1.66 & 1.72 & 1.76 & 1.79 & 1.81 & 1.87 & 1.92 & 1.97 \\
\hline & & SSJPM & 1.52 & 1.57 & 1.64 & 1.70 & 1.80 & 1.91 & 1.98 & 2.03 & 2.25 & 2.45 & 2.67 \\
\hline & & $97.5 \%$ & & & & & & & & & & & \\
\hline & & GPD $2.5 \%$ & 1.42 & 1.46 & 1.48 & 1.50 & 1.51 & 1.51 & 1.52 & 1.52 & 1.52 & 1.52 & 1.52 \\
\hline & & GPD $50 \%$ & 1.45 & 1.50 & 1.55 & 1.58 & 1.62 & 1.64 & 1.65 & 1.66 & 1.68 & 1.69 & 1.70 \\
\hline & & GPD 97.5\% & 1.49 & 1.54 & 1.60 & 1.64 & 1.70 & 1.75 & 1.78 & 1.80 & 1.86 & 1.91 & 1.96 \\
\hline \multirow[t]{7}{*}{2} & \multirow[t]{7}{*}{ Whangaroa } & SSJPM $2.5 \%$ & 1.38 & 1.41 & 1.46 & 1.49 & 1.52 & 1.55 & 1.57 & 1.58 & 1.61 & 1.64 & 1.66 \\
\hline & & SSJPM 50\% & 1.39 & 1.43 & 1.48 & 1.51 & 1.57 & 1.61 & 1.63 & 1.65 & 1.70 & 1.74 & 1.78 \\
\hline & & SSJPM & 1.40 & 1.45 & 1.51 & 1.56 & 1.65 & 1.73 & 1.78 & 1.81 & 1.94 & 2.07 & 2.20 \\
\hline & & $97.5 \%$ & & & & & & & & & & & \\
\hline & & GPD $2.5 \%$ & 1.34 & 1.36 & 1.37 & 1.38 & 1.38 & 1.38 & 1.38 & 1.39 & 1.39 & 1.39 & 1.39 \\
\hline & & GPD 50\% & 1.38 & 1.42 & 1.46 & 1.49 & 1.51 & 1.53 & 1.54 & 1.54 & 1.55 & 1.56 & 1.57 \\
\hline & & GPD 97.5\% & 1.43 & 1.47 & 1.51 & 1.54 & 1.59 & 1.62 & 1.64 & 1.65 & 1.69 & 1.72 & 1.75 \\
\hline \multirow[t]{7}{*}{3} & \multirow[t]{7}{*}{ Opua } & SSJPM $2.5 \%$ & 1.42 & 1.45 & 1.50 & 1.53 & 1.57 & 1.60 & 1.62 & 1.63 & 1.67 & 1.69 & 1.72 \\
\hline & & SSJPM 50\% & 1.42 & 1.46 & 1.51 & 1.54 & 1.59 & 1.62 & 1.64 & 1.65 & 1.69 & 1.72 & 1.76 \\
\hline & & SSJPM & 1.43 & 1.47 & 1.52 & 1.56 & 1.61 & 1.65 & 1.68 & 1.70 & 1.76 & 1.80 & 1.85 \\
\hline & & $97.5 \%$ & & & & & & & & & & & \\
\hline & & GPD $2.5 \%$ & 1.38 & 1.42 & 1.46 & 1.49 & 1.52 & 1.54 & 1.55 & 1.55 & 1.57 & 1.58 & 1.58 \\
\hline & & GPD 50\% & 1.40 & 1.45 & 1.51 & 1.55 & 1.60 & 1.63 & 1.65 & 1.66 & 1.70 & 1.72 & 1.74 \\
\hline & & GPD 97.5\% & 1.43 & 1.48 & 1.55 & 1.61 & 1.68 & 1.74 & 1.78 & 1.80 & 1.88 & 1.94 & 2.00 \\
\hline \multirow[t]{7}{*}{4} & \multirow[t]{7}{*}{ Marsden Point } & SSJPM 2.5\% & 1.51 & 1.55 & 1.61 & 1.65 & 1.70 & 1.75 & 1.77 & 1.79 & 1.84 & 1.87 & 1.90 \\
\hline & & SSJPM 50\% & 1.51 & 1.56 & 1.62 & 1.67 & 1.73 & 1.78 & 1.81 & 1.83 & 1.89 & 1.94 & 1.99 \\
\hline & & SSJPM & 1.52 & 1.57 & 1.63 & 1.68 & 1.76 & 1.83 & 1.88 & 1.91 & 2.03 & 2.13 & 2.24 \\
\hline & & $97.5 \%$ & & & & & & & & & & & \\
\hline & & GPD $2.5 \%$ & 1.45 & 1.50 & 1.56 & 1.60 & 1.65 & 1.68 & 1.70 & 1.71 & 1.74 & 1.77 & 1.79 \\
\hline & & GPD 50\% & 1.47 & 1.52 & 1.59 & 1.65 & 1.72 & 1.77 & 1.80 & 1.82 & 1.89 & 1.93 & 1.98 \\
\hline & & GPD $97.5 \%$ & 1.49 & 1.55 & 1.63 & 1.70 & 1.80 & 1.88 & 1.93 & 1.96 & 2.08 & 2.17 & 2.27 \\
\hline
\end{tabular}




\begin{tabular}{|c|c|c|c|c|c|c|c|c|c|c|c|c|c|}
\hline \multirow{2}{*}{ 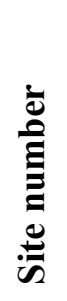 } & \multirow{2}{*}{ 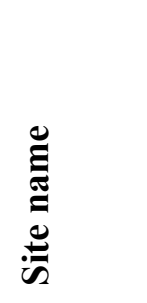 } & \multirow{2}{*}{ 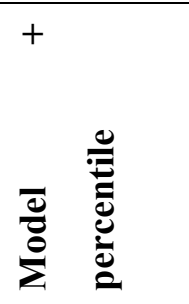 } & \multicolumn{11}{|c|}{ Return period (years) } \\
\hline & & & 1 & 2 & 5 & 10 & 25 & 50 & 75 & 100 & 250 & 500 & 1000 \\
\hline \multirow[t]{7}{*}{5} & \multirow[t]{7}{*}{ Auckland } & SSJPM $2.5 \%$ & 1.91 & 1.96 & 2.01 & 2.06 & 2.11 & 2.15 & 2.18 & 2.20 & 2.25 & 2.29 & 2.33 \\
\hline & & SSJPM 50\% & 1.91 & 1.96 & 2.02 & 2.06 & 2.12 & 2.17 & 2.20 & 2.22 & 2.29 & 2.34 & 2.39 \\
\hline & & SSJPM & 1.91 & 1.96 & 2.02 & 2.07 & 2.13 & 2.19 & 2.22 & 2.24 & 2.32 & 2.37 & 2.43 \\
\hline & & $97.5 \%$ & & & & & & & & & & & \\
\hline & & GPD $2.5 \%$ & 1.85 & 1.90 & 1.96 & 2.00 & 2.05 & 2.08 & 2.09 & 2.10 & 2.13 & 2.15 & 2.17 \\
\hline & & GPD $50 \%$ & 1.86 & 1.92 & 1.99 & 2.04 & 2.09 & 2.13 & 2.16 & 2.17 & 2.21 & 2.25 & 2.27 \\
\hline & & GPD $97.5 \%$ & 1.87 & 1.93 & 2.01 & 2.07 & 2.14 & 2.19 & 2.22 & 2.24 & 2.30 & 2.35 & 2.39 \\
\hline \multirow[t]{7}{*}{6} & \multirow[t]{7}{*}{ Whitianga } & SSJPM $2.5 \%$ & 1.14 & 1.17 & 1.21 & 1.25 & 1.29 & 1.32 & 1.34 & 1.35 & 1.38 & 1.40 & 1.42 \\
\hline & & SSJPM 50\% & 1.14 & 1.18 & 1.24 & 1.28 & 1.33 & 1.37 & 1.40 & 1.41 & 1.47 & 1.51 & 1.55 \\
\hline & & SSJPM & 1.15 & 1.20 & 1.26 & 1.31 & 1.40 & 1.47 & 1.51 & 1.54 & 1.65 & 1.75 & 1.86 \\
\hline & & $97.5 \%$ & & & & & & & & & & & \\
\hline & & GPD $2.5 \%$ & 1.09 & 1.13 & 1.17 & 1.20 & 1.23 & 1.25 & 1.26 & 1.26 & 1.28 & 1.29 & 1.30 \\
\hline & & GPD $50 \%$ & 1.11 & 1.16 & 1.21 & 1.25 & 1.30 & 1.33 & 1.35 & 1.36 & 1.39 & 1.42 & 1.44 \\
\hline & & GPD $97.5 \%$ & 1.14 & 1.19 & 1.25 & 1.30 & 1.38 & 1.43 & 1.47 & 1.50 & 1.58 & 1.65 & 1.72 \\
\hline \multirow[t]{7}{*}{7} & \multirow[t]{7}{*}{ Tararu } & SSJPM $2.5 \%$ & 2.03 & 2.08 & 2.13 & 2.17 & 2.23 & 2.27 & 2.30 & 2.31 & 2.36 & 2.40 & 2.43 \\
\hline & & SSJPM 50\% & 2.04 & 2.09 & 2.15 & 2.19 & 2.26 & 2.31 & 2.35 & 2.37 & 2.43 & 2.47 & 2.51 \\
\hline & & SSJPM & 2.05 & 2.09 & 2.16 & 2.21 & 2.29 & 2.35 & 2.39 & 2.41 & 2.48 & 2.53 & 2.58 \\
\hline & & $97.5 \%$ & & & & & & & & & & & \\
\hline & & GPD $2.5 \%$ & 1.96 & 2.01 & 2.07 & 2.11 & 2.15 & 2.18 & 2.19 & 2.20 & 2.23 & 2.25 & 2.27 \\
\hline & & GPD $50 \%$ & 1.99 & 2.05 & 2.13 & 2.20 & 2.28 & 2.35 & 2.39 & 2.41 & 2.50 & 2.57 & 2.63 \\
\hline & & GPD $97.5 \%$ & 2.02 & 2.09 & 2.20 & 2.29 & 2.44 & 2.56 & 2.64 & 2.70 & 2.91 & 3.08 & 3.27 \\
\hline \multirow[t]{7}{*}{8} & \multirow[t]{7}{*}{ Tauranga } & SSJPM $2.5 \%$ & 1.17 & 1.20 & 1.24 & 1.27 & 1.31 & 1.33 & 1.35 & 1.36 & 1.40 & 1.42 & 1.44 \\
\hline & & SSJPM 50\% & 1.17 & 1.20 & 1.25 & 1.28 & 1.33 & 1.36 & 1.38 & 1.39 & 1.43 & 1.46 & 1.49 \\
\hline & & SSJPM & 1.18 & 1.21 & 1.26 & 1.30 & 1.35 & 1.40 & 1.42 & 1.44 & 1.50 & 1.54 & 1.59 \\
\hline & & $97.5 \%$ & & & & & & & & & & & \\
\hline & & GPD $2.5 \%$ & 1.12 & 1.15 & 1.19 & 1.21 & 1.23 & 1.25 & 1.25 & 1.26 & 1.27 & 1.28 & 1.28 \\
\hline & & GPD 50\% & 1.14 & 1.17 & 1.22 & 1.24 & 1.28 & 1.30 & 1.31 & 1.31 & 1.33 & 1.35 & 1.36 \\
\hline & & GPD $97.5 \%$ & 1.15 & 1.19 & 1.24 & 1.28 & 1.32 & 1.35 & 1.37 & 1.38 & 1.42 & 1.44 & 1.47 \\
\hline \multirow[t]{2}{*}{9} & \multirow[t]{2}{*}{ Moturiki } & SSJPM $2.5 \%$ & 1.23 & 1.26 & 1.30 & 1.34 & 1.38 & 1.41 & 1.43 & 1.44 & 1.48 & 1.51 & 1.54 \\
\hline & & SSJPM 50\% & 1.23 & 1.27 & 1.31 & 1.35 & 1.40 & 1.44 & 1.46 & 1.48 & 1.54 & 1.57 & 1.60 \\
\hline
\end{tabular}




\begin{tabular}{|c|c|c|c|c|c|c|c|c|c|c|c|c|c|}
\hline \multirow{2}{*}{ 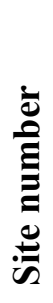 } & \multirow{2}{*}{$\begin{array}{l}\stackrel{\Xi}{\Xi} \\
\stackrel{\Xi}{\Xi} \\
\text { 导 }\end{array}$} & \multirow{2}{*}{ 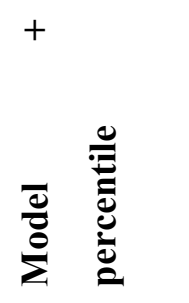 } & \multicolumn{11}{|c|}{ Return period (years) } \\
\hline & & & 1 & 2 & 5 & 10 & 25 & 50 & 75 & 100 & 250 & 500 & 1000 \\
\hline & & $\begin{array}{l}\text { SSJPM } \\
97.5 \%\end{array}$ & 1.24 & 1.27 & 1.32 & 1.36 & 1.42 & 1.47 & 1.50 & 1.52 & 1.58 & 1.62 & 1.67 \\
\hline & & GPD $2.5 \%$ & 1.19 & 1.23 & 1.27 & 1.30 & 1.34 & 1.36 & 1.38 & 1.38 & 1.41 & 1.43 & 1.44 \\
\hline & & GPD $50 \%$ & 1.20 & 1.25 & 1.30 & 1.34 & 1.39 & 1.43 & 1.45 & 1.47 & 1.51 & 1.55 & 1.58 \\
\hline & & GPD $97.5 \%$ & 1.22 & 1.27 & 1.33 & 1.38 & 1.46 & 1.51 & 1.55 & 1.57 & 1.65 & 1.72 & 1.78 \\
\hline \multirow[t]{7}{*}{10} & Gisborne & SSJPM $2.5 \%$ & 1.10 & 1.13 & 1.17 & 1.20 & 1.23 & 1.25 & 1.26 & 1.27 & 1.29 & 1.30 & 1.31 \\
\hline & & SSJPM 50\% & 1.12 & 1.15 & 1.19 & 1.22 & 1.25 & 1.27 & 1.29 & 1.29 & 1.32 & 1.33 & 1.34 \\
\hline & & SSJPM & 1.13 & 1.16 & 1.21 & 1.24 & 1.27 & 1.29 & 1.31 & 1.32 & 1.35 & 1.37 & 1.39 \\
\hline & & $97.5 \%$ & & & & & & & & & & & \\
\hline & & GPD $2.5 \%$ & 1.06 & 1.09 & 1.13 & 1.15 & 1.18 & 1.19 & 1.19 & 1.19 & 1.20 & 1.20 & 1.20 \\
\hline & & GPD 50\% & 1.09 & 1.13 & 1.18 & 1.21 & 1.24 & 1.27 & 1.28 & 1.29 & 1.32 & 1.34 & 1.35 \\
\hline & & GPD $97.5 \%$ & 1.15 & 1.17 & 1.23 & 1.31 & 1.44 & 1.55 & 1.64 & 1.70 & 1.92 & 2.13 & 2.39 \\
\hline \multirow[t]{7}{*}{11} & Wellington & SSJPM $2.5 \%$ & 0.96 & 0.99 & 1.04 & 1.07 & 1.11 & 1.15 & 1.16 & 1.17 & 1.21 & 1.23 & 1.25 \\
\hline & & SSJPM 50\% & 0.96 & 1.00 & 1.05 & 1.08 & 1.13 & 1.17 & 1.19 & 1.20 & 1.24 & 1.26 & 1.29 \\
\hline & & SSJPM & 0.97 & 1.01 & 1.05 & 1.09 & 1.14 & 1.18 & 1.20 & 1.21 & 1.25 & 1.28 & 1.30 \\
\hline & & $97.5 \%$ & & & & & & & & & & & \\
\hline & & GPD $2.5 \%$ & 0.94 & 0.97 & 1.01 & 1.04 & 1.06 & 1.08 & 1.09 & 1.09 & 1.11 & 1.12 & 1.12 \\
\hline & & GPD 50\% & 0.95 & 0.99 & 1.03 & 1.06 & 1.10 & 1.12 & 1.13 & 1.14 & 1.16 & 1.18 & 1.19 \\
\hline & & GPD 97.5\% & 0.96 & 1.00 & 1.05 & 1.08 & 1.13 & 1.16 & 1.18 & 1.19 & 1.22 & 1.25 & 1.27 \\
\hline \multirow[t]{7}{*}{12} & Kaikoura & SSJPM $2.5 \%$ & 1.17 & 1.20 & 1.24 & 1.26 & 1.29 & 1.31 & 1.33 & 1.33 & 1.36 & 1.37 & 1.38 \\
\hline & & SSJPM 50\% & 1.18 & 1.21 & 1.25 & 1.27 & 1.30 & 1.33 & 1.34 & 1.35 & 1.37 & 1.39 & 1.41 \\
\hline & & SSJPM & 1.18 & 1.21 & 1.25 & 1.28 & 1.32 & 1.34 & 1.36 & 1.37 & 1.40 & 1.42 & 1.44 \\
\hline & & $97.5 \%$ & & & & & & & & & & & \\
\hline & & GPD $2.5 \%$ & 1.13 & 1.16 & 1.19 & 1.21 & 1.22 & 1.24 & 1.24 & 1.24 & 1.25 & 1.26 & 1.26 \\
\hline & & GPD 50\% & 1.15 & 1.19 & 1.24 & 1.28 & 1.33 & 1.36 & 1.37 & 1.38 & 1.42 & 1.44 & 1.47 \\
\hline & & GPD $97.5 \%$ & 1.18 & 1.23 & 1.30 & 1.35 & 1.43 & 1.50 & 1.55 & 1.58 & 1.68 & 1.77 & 1.87 \\
\hline \multirow[t]{4}{*}{13} & Sumner & SSJPM $2.5 \%$ & 1.46 & 1.51 & 1.55 & 1.59 & 1.63 & 1.66 & 1.68 & 1.69 & 1.73 & 1.75 & 1.77 \\
\hline & & SSJPM 50\% & 1.47 & 1.51 & 1.56 & 1.60 & 1.64 & 1.67 & 1.69 & 1.70 & 1.74 & 1.77 & 1.79 \\
\hline & & $\begin{array}{l}\text { SSJPM } \\
97.5 \%\end{array}$ & 1.47 & 1.52 & 1.57 & 1.61 & 1.65 & 1.69 & 1.71 & 1.72 & 1.76 & 1.79 & 1.82 \\
\hline & & GPD $2.5 \%$ & 1.41 & 1.46 & 1.51 & 1.54 & 1.57 & 1.60 & 1.61 & 1.61 & 1.63 & 1.65 & 1.66 \\
\hline
\end{tabular}




\begin{tabular}{|c|c|c|c|c|c|c|c|c|c|c|c|c|c|}
\hline \multirow{2}{*}{ 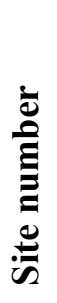 } & \multirow{2}{*}{$\begin{array}{l}\stackrel{\mathscr{\Xi}}{\Xi} \\
\stackrel{\Xi}{\Xi} \\
\text { : }\end{array}$} & \multirow{2}{*}{ 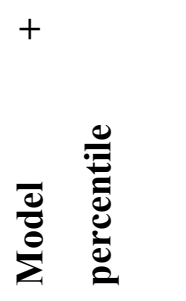 } & \multicolumn{11}{|c|}{ Return period (years) } \\
\hline & & & 1 & 2 & 5 & 10 & 25 & 50 & 75 & 100 & 250 & 500 & 1000 \\
\hline & & GPD $50 \%$ & 1.44 & 1.49 & 1.55 & 1.60 & 1.66 & 1.70 & 1.73 & 1.74 & 1.79 & 1.83 & 1.86 \\
\hline & & GPD $97.5 \%$ & 1.46 & 1.52 & 1.60 & 1.67 & 1.76 & 1.84 & 1.89 & 1.92 & 2.04 & 2.14 & 2.24 \\
\hline \multirow[t]{6}{*}{14} & Lyttelton & SSJPM $2.5 \%$ & 1.53 & 1.57 & 1.62 & 1.66 & 1.71 & 1.74 & 1.76 & 1.77 & 1.81 & 1.84 & 1.87 \\
\hline & & SSJPM 50\% & 1.53 & 1.57 & 1.63 & 1.67 & 1.71 & 1.75 & 1.77 & 1.78 & 1.83 & 1.86 & 1.89 \\
\hline & & $\begin{array}{l}\text { SSJPM } \\
97.5 \%\end{array}$ & 1.53 & 1.58 & 1.63 & 1.67 & 1.73 & 1.76 & 1.78 & 1.80 & 1.85 & 1.88 & 1.92 \\
\hline & & GPD $2.5 \%$ & 1.48 & 1.52 & 1.57 & 1.59 & 1.62 & 1.64 & 1.65 & 1.65 & 1.66 & 1.67 & 1.68 \\
\hline & & GPD $50 \%$ & 1.49 & 1.54 & 1.59 & 1.63 & 1.67 & 1.69 & 1.71 & 1.72 & 1.74 & 1.76 & 1.77 \\
\hline & & GPD $97.5 \%$ & 1.50 & 1.55 & 1.61 & 1.65 & 1.70 & 1.73 & 1.75 & 1.76 & 1.80 & 1.82 & 1.84 \\
\hline \multirow[t]{6}{*}{15} & Timaru & SSJPM $2.5 \%$ & 1.39 & 1.43 & 1.48 & 1.51 & 1.55 & 1.58 & 1.60 & 1.61 & 1.64 & 1.66 & 1.68 \\
\hline & & SSJPM 50\% & 1.40 & 1.44 & 1.49 & 1.53 & 1.58 & 1.62 & 1.64 & 1.65 & 1.69 & 1.72 & 1.74 \\
\hline & & $\begin{array}{l}\text { SSJPM } \\
97.5 \%\end{array}$ & 1.41 & 1.45 & 1.51 & 1.55 & 1.61 & 1.65 & 1.67 & 1.68 & 1.73 & 1.76 & 1.79 \\
\hline & & GPD $2.5 \%$ & 1.36 & 1.39 & 1.42 & 1.44 & 1.46 & 1.46 & 1.47 & 1.47 & 1.48 & 1.48 & 1.48 \\
\hline & & GPD $50 \%$ & 1.38 & 1.43 & 1.48 & 1.52 & 1.56 & 1.58 & 1.60 & 1.61 & 1.63 & 1.65 & 1.66 \\
\hline & & GPD $97.5 \%$ & 1.41 & 1.46 & 1.52 & 1.57 & 1.63 & 1.68 & 1.71 & 1.72 & 1.78 & 1.82 & 1.87 \\
\hline \multirow[t]{6}{*}{16} & Dunedin & SSJPM $2.5 \%$ & 1.46 & 1.50 & 1.56 & 1.60 & 1.64 & 1.68 & 1.70 & 1.71 & 1.75 & 1.78 & 1.80 \\
\hline & & SSJPM 50\% & 1.47 & 1.51 & 1.56 & 1.60 & 1.65 & 1.68 & 1.70 & 1.72 & 1.76 & 1.79 & 1.82 \\
\hline & & $\begin{array}{l}\text { SSJPM } \\
97.5 \%\end{array}$ & 1.47 & 1.51 & 1.57 & 1.61 & 1.66 & 1.69 & 1.71 & 1.73 & 1.77 & 1.80 & 1.83 \\
\hline & & GPD $2.5 \%$ & 1.41 & 1.45 & 1.51 & 1.55 & 1.59 & 1.62 & 1.64 & 1.65 & 1.68 & 1.70 & 1.72 \\
\hline & & GPD $50 \%$ & 1.42 & 1.47 & 1.53 & 1.58 & 1.63 & 1.67 & 1.69 & 1.71 & 1.76 & 1.79 & 1.82 \\
\hline & & GPD $97.5 \%$ & 1.43 & 1.48 & 1.55 & 1.61 & 1.68 & 1.73 & 1.77 & 1.79 & 1.86 & 1.92 & 1.97 \\
\hline \multirow[t]{6}{*}{17} & Green Island & SSJPM $2.5 \%$ & 1.34 & 1.38 & 1.42 & 1.45 & 1.49 & 1.52 & 1.54 & 1.55 & 1.58 & 1.61 & 1.63 \\
\hline & & SSJPM 50\% & 1.35 & 1.38 & 1.43 & 1.47 & 1.51 & 1.54 & 1.56 & 1.57 & 1.61 & 1.63 & 1.66 \\
\hline & & $\begin{array}{l}\text { SSJPM } \\
97.5 \%\end{array}$ & 1.35 & 1.39 & 1.45 & 1.48 & 1.53 & 1.57 & 1.59 & 1.60 & 1.65 & 1.68 & 1.72 \\
\hline & & GPD $2.5 \%$ & 1.30 & 1.33 & 1.35 & 1.37 & 1.38 & 1.38 & 1.38 & 1.39 & 1.39 & 1.39 & 1.39 \\
\hline & & GPD 50\% & 1.32 & 1.36 & 1.39 & 1.42 & 1.44 & 1.45 & 1.46 & 1.46 & 1.47 & 1.48 & 1.49 \\
\hline & & GPD $97.5 \%$ & 1.35 & 1.38 & 1.42 & 1.45 & 1.48 & 1.50 & 1.51 & 1.52 & 1.55 & 1.56 & 1.58 \\
\hline 18 & Invercargill & SSJPM $2.5 \%$ & 1.69 & 1.76 & 1.86 & 1.92 & 2.00 & 2.07 & 2.11 & 2.13 & 2.20 & 2.25 & 2.30 \\
\hline
\end{tabular}




\begin{tabular}{|c|c|c|c|c|c|c|c|c|c|c|c|c|c|}
\hline \multirow{2}{*}{ 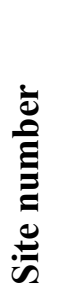 } & \multirow{2}{*}{$\begin{array}{l}\stackrel{\mathscr{\Xi}}{\Xi} \\
\stackrel{\Xi}{\Xi} \\
\stackrel{\Xi}{\Xi}\end{array}$} & \multirow{2}{*}{ 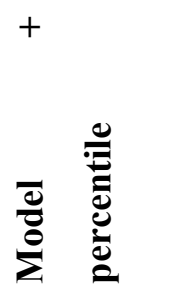 } & \multicolumn{11}{|c|}{ Return period (years) } \\
\hline & & & 1 & 2 & 5 & 10 & 25 & 50 & 75 & 100 & 250 & 500 & 1000 \\
\hline & & SSJPM 50\% & 1.71 & 1.79 & 1.89 & 1.97 & 2.07 & 2.15 & 2.19 & 2.22 & 2.30 & 2.37 & 2.44 \\
\hline & & $\begin{array}{l}\text { SSJPM } \\
97.5 \%\end{array}$ & 1.73 & 1.82 & 1.94 & 2.04 & 2.18 & 2.28 & 2.34 & 2.39 & 2.53 & 2.64 & 2.76 \\
\hline & & GPD $2.5 \%$ & 1.66 & 1.73 & 1.81 & 1.86 & 1.90 & 1.93 & 1.95 & 1.96 & 1.99 & 2.00 & 2.02 \\
\hline & & GPD $50 \%$ & 1.70 & 1.78 & 1.89 & 1.96 & 2.05 & 2.12 & 2.15 & 2.18 & 2.25 & 2.30 & 2.35 \\
\hline & & GPD 97.5\% & 1.74 & 1.83 & 1.96 & 2.06 & 2.20 & 2.31 & 2.38 & 2.42 & 2.58 & 2.69 & 2.82 \\
\hline \multirow[t]{6}{*}{19} & Dog Island & SSJPM $2.5 \%$ & 1.59 & 1.63 & 1.68 & 1.71 & 1.75 & 1.78 & 1.79 & 1.80 & 1.83 & 1.86 & 1.88 \\
\hline & & SSJPM 50\% & 1.59 & 1.63 & 1.68 & 1.72 & 1.76 & 1.79 & 1.81 & 1.82 & 1.86 & 1.88 & 1.90 \\
\hline & & $\begin{array}{l}\text { SSJPM } \\
97.5 \%\end{array}$ & 1.60 & 1.64 & 1.70 & 1.73 & 1.78 & 1.81 & 1.83 & 1.85 & 1.89 & 1.92 & 1.95 \\
\hline & & GPD $2.5 \%$ & 1.53 & 1.57 & 1.61 & 1.64 & 1.66 & 1.67 & 1.68 & 1.68 & 1.69 & 1.69 & 1.70 \\
\hline & & GPD 50\% & 1.55 & 1.59 & 1.64 & 1.67 & 1.70 & 1.72 & 1.73 & 1.74 & 1.75 & 1.76 & 1.77 \\
\hline & & GPD 97.5\% & 1.58 & 1.62 & 1.67 & 1.70 & 1.75 & 1.79 & 1.81 & 1.83 & 1.87 & 1.90 & 1.93 \\
\hline \multirow[t]{6}{*}{20} & Jackson Bay & SSJPM $2.5 \%$ & 1.55 & 1.60 & 1.66 & 1.70 & 1.75 & 1.78 & 1.80 & 1.82 & 1.85 & 1.88 & 1.91 \\
\hline & & SSJPM 50\% & 1.56 & 1.61 & 1.67 & 1.72 & 1.77 & 1.81 & 1.83 & 1.84 & 1.89 & 1.92 & 1.95 \\
\hline & & $\begin{array}{l}\text { SSJPM } \\
97.5 \%\end{array}$ & 1.57 & 1.62 & 1.69 & 1.73 & 1.79 & 1.83 & 1.85 & 1.87 & 1.92 & 1.95 & 1.99 \\
\hline & & GPD $2.5 \%$ & 1.52 & 1.58 & 1.63 & 1.66 & 1.69 & 1.71 & 1.72 & 1.73 & 1.74 & 1.75 & 1.76 \\
\hline & & GPD $50 \%$ & 1.56 & 1.63 & 1.72 & 1.79 & 1.87 & 1.93 & 1.96 & 1.99 & 2.05 & 2.10 & 2.15 \\
\hline & & GPD 97.5\% & 1.61 & 1.69 & 1.81 & 1.91 & 2.06 & 2.19 & 2.28 & 2.34 & 2.56 & 2.74 & 2.94 \\
\hline \multirow[t]{6}{*}{21} & Charleston & SSJPM 2.5\% & 1.94 & 1.99 & 2.05 & 2.09 & 2.15 & 2.18 & 2.20 & 2.22 & 2.26 & 2.28 & 2.31 \\
\hline & & SSJPM 50\% & 1.94 & 2.00 & 2.06 & 2.11 & 2.16 & 2.20 & 2.23 & 2.24 & 2.29 & 2.32 & 2.35 \\
\hline & & $\begin{array}{l}\text { SSJPM } \\
97.5 \%\end{array}$ & 1.95 & 2.01 & 2.08 & 2.12 & 2.18 & 2.23 & 2.25 & 2.27 & 2.33 & 2.37 & 2.41 \\
\hline & & GPD $2.5 \%$ & 1.86 & 1.90 & 1.95 & 1.97 & 1.99 & 2.01 & 2.01 & 2.01 & 2.02 & 2.03 & 2.03 \\
\hline & & GPD 50\% & 1.89 & 1.96 & 2.05 & 2.12 & 2.20 & 2.26 & 2.29 & 2.32 & 2.39 & 2.44 & 2.49 \\
\hline & & GPD 97.5\% & 1.93 & 2.02 & 2.15 & 2.25 & 2.40 & 2.52 & 2.59 & 2.65 & 2.84 & 2.99 & 3.15 \\
\hline \multirow[t]{3}{*}{22} & Nelson & SSJPM 2.5\% & 2.37 & 2.42 & 2.49 & 2.53 & 2.58 & 2.62 & 2.64 & 2.66 & 2.71 & 2.75 & 2.78 \\
\hline & & SSJPM 50\% & 2.38 & 2.43 & 2.49 & 2.54 & 2.59 & 2.64 & 2.66 & 2.68 & 2.74 & 2.78 & 2.83 \\
\hline & & $\begin{array}{l}\text { SSJPM } \\
97.5 \%\end{array}$ & 2.38 & 2.44 & 2.50 & 2.55 & 2.61 & 2.66 & 2.69 & 2.72 & 2.80 & 2.88 & 2.95 \\
\hline
\end{tabular}




\begin{tabular}{|c|c|c|c|c|c|c|c|c|c|c|c|c|c|}
\hline \multirow{2}{*}{ 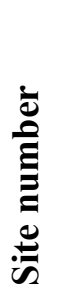 } & \multirow{2}{*}{$\begin{array}{l}\stackrel{\mathscr{\Xi}}{\Xi} \\
\stackrel{\Xi}{\Xi} \\
\stackrel{\Xi}{\Xi}\end{array}$} & \multirow{2}{*}{ 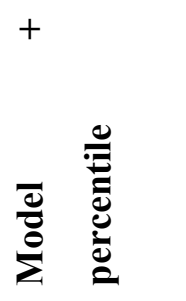 } & \multicolumn{11}{|c|}{ Return period (years) } \\
\hline & & & 1 & 2 & 5 & 10 & 25 & 50 & 75 & 100 & 250 & 500 & 1000 \\
\hline & & GPD $2.5 \%$ & 2.32 & 2.36 & 2.39 & 2.41 & 2.43 & 2.44 & 2.44 & 2.44 & 2.45 & 2.45 & 2.45 \\
\hline & & GPD 50\% & 2.34 & 2.39 & 2.44 & 2.47 & 2.51 & 2.53 & 2.54 & 2.55 & 2.57 & 2.59 & 2.60 \\
\hline & & GPD $97.5 \%$ & 2.36 & 2.42 & 2.48 & 2.52 & 2.57 & 2.60 & 2.62 & 2.63 & 2.67 & 2.70 & 2.72 \\
\hline \multirow[t]{6}{*}{23} & Kapiti & SSJPM $2.5 \%$ & 1.24 & 1.27 & 1.32 & 1.35 & 1.39 & 1.42 & 1.43 & 1.44 & 1.47 & 1.49 & 1.51 \\
\hline & & SSJPM 50\% & 1.25 & 1.28 & 1.33 & 1.37 & 1.41 & 1.44 & 1.46 & 1.47 & 1.50 & 1.53 & 1.55 \\
\hline & & $\begin{array}{l}\text { SSJPM } \\
97.5 \%\end{array}$ & 1.25 & 1.29 & 1.35 & 1.38 & 1.43 & 1.47 & 1.49 & 1.51 & 1.56 & 1.59 & 1.63 \\
\hline & & GPD $2.5 \%$ & 1.20 & 1.23 & 1.26 & 1.27 & 1.28 & 1.29 & 1.29 & 1.29 & 1.30 & 1.30 & 1.30 \\
\hline & & GPD $50 \%$ & 1.23 & 1.26 & 1.30 & 1.32 & 1.35 & 1.36 & 1.37 & 1.37 & 1.38 & 1.39 & 1.40 \\
\hline & & GPD 97.5\% & 1.25 & 1.29 & 1.33 & 1.35 & 1.39 & 1.41 & 1.43 & 1.43 & 1.46 & 1.48 & 1.49 \\
\hline \multirow[t]{6}{*}{24} & Taranaki & SSJPM $2.5 \%$ & 2.07 & 2.11 & 2.17 & 2.21 & 2.26 & 2.29 & 2.31 & 2.32 & 2.36 & 2.38 & 2.41 \\
\hline & & SSJPM 50\% & 2.07 & 2.12 & 2.18 & 2.22 & 2.26 & 2.30 & 2.32 & 2.33 & 2.37 & 2.40 & 2.43 \\
\hline & & $\begin{array}{l}\text { SSJPM } \\
97.5 \%\end{array}$ & 2.08 & 2.12 & 2.18 & 2.22 & 2.27 & 2.31 & 2.33 & 2.34 & 2.39 & 2.42 & 2.44 \\
\hline & & GPD $2.5 \%$ & 1.99 & 2.05 & 2.11 & 2.14 & 2.18 & 2.20 & 2.21 & 2.22 & 2.24 & 2.25 & 2.26 \\
\hline & & GPD $50 \%$ & 2.02 & 2.08 & 2.15 & 2.20 & 2.26 & 2.30 & 2.32 & 2.34 & 2.38 & 2.41 & 2.44 \\
\hline & & GPD $97.5 \%$ & 2.05 & 2.11 & 2.20 & 2.26 & 2.35 & 2.42 & 2.46 & 2.49 & 2.58 & 2.65 & 2.72 \\
\hline \multirow[t]{6}{*}{25} & Kawhia & SSJPM $2.5 \%$ & 2.14 & 2.19 & 2.26 & 2.31 & 2.37 & 2.41 & 2.43 & 2.44 & 2.49 & 2.52 & 2.54 \\
\hline & & SSJPM 50\% & 2.17 & 2.24 & 2.33 & 2.40 & 2.50 & 2.58 & 2.62 & 2.66 & 2.76 & 2.82 & 2.88 \\
\hline & & $\begin{array}{l}\text { SSJPM } \\
97.5 \%\end{array}$ & 2.20 & 2.29 & 2.41 & 2.51 & 2.66 & 2.78 & 2.86 & 2.91 & 3.10 & 3.26 & 3.43 \\
\hline & & GPD $2.5 \%$ & 2.02 & 2.06 & 2.11 & 2.15 & 2.18 & 2.20 & 2.21 & 2.22 & 2.24 & 2.25 & 2.26 \\
\hline & & GPD $50 \%$ & 2.06 & 2.13 & 2.22 & 2.29 & 2.38 & 2.45 & 2.49 & 2.51 & 2.60 & 2.67 & 2.73 \\
\hline & & GPD $97.5 \%$ & 2.12 & 2.20 & 2.33 & 2.44 & 2.63 & 2.80 & 2.92 & 3.00 & 3.33 & 3.61 & 3.94 \\
\hline \multirow[t]{6}{*}{26} & Raglan & SSJPM $2.5 \%$ & 2.05 & 2.12 & 2.20 & 2.26 & 2.33 & 2.38 & 2.41 & 2.43 & 2.49 & 2.53 & 2.57 \\
\hline & & SSJPM 50\% & 2.08 & 2.16 & 2.27 & 2.35 & 2.47 & 2.57 & 2.63 & 2.67 & 2.80 & 2.91 & 3.01 \\
\hline & & $\begin{array}{l}\text { SSJPM } \\
97.5 \%\end{array}$ & 2.12 & 2.21 & 2.36 & 2.49 & 2.69 & 2.87 & 2.99 & 3.08 & 3.40 & 3.69 & 4.00 \\
\hline & & GPD $2.5 \%$ & 1.96 & 2.02 & 2.09 & 2.14 & 2.20 & 2.23 & 2.25 & 2.26 & 2.29 & 2.31 & 2.32 \\
\hline & & GPD 50\% & 2.03 & 2.12 & 2.24 & 2.34 & 2.46 & 2.56 & 2.61 & 2.65 & 2.79 & 2.89 & 2.99 \\
\hline & & GPD 97.5\% & 2.12 & 2.22 & 2.40 & 2.58 & 2.93 & 3.26 & 3.51 & 3.69 & 4.47 & 5.17 & 6.11 \\
\hline
\end{tabular}




\begin{tabular}{|c|c|c|c|c|c|c|c|c|c|c|c|c|c|}
\hline \multirow{2}{*}{ 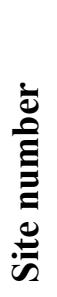 } & \multirow{2}{*}{ 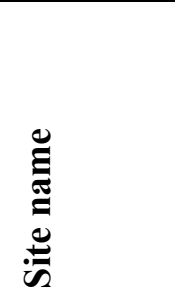 } & \multirow{2}{*}{ 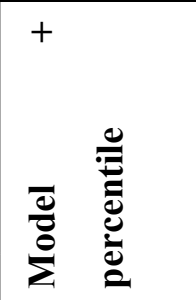 } & \multicolumn{11}{|c|}{ Return period (years) } \\
\hline & & & 1 & 2 & 5 & 10 & 25 & 50 & 75 & 100 & 250 & 500 & 1000 \\
\hline \multirow[t]{6}{*}{27} & Onehunga & SSJPM $2.5 \%$ & 2.24 & 2.29 & 2.36 & 2.40 & 2.45 & 2.49 & 2.51 & 2.53 & 2.57 & 2.60 & 2.63 \\
\hline & & SSJPM 50\% & 2.25 & 2.30 & 2.37 & 2.42 & 2.48 & 2.52 & 2.55 & 2.57 & 2.62 & 2.66 & 2.70 \\
\hline & & $\begin{array}{l}\text { SSJPM } \\
97.5 \%\end{array}$ & 2.26 & 2.31 & 2.38 & 2.44 & 2.51 & 2.57 & 2.61 & 2.64 & 2.74 & 2.82 & 2.91 \\
\hline & & GPD $2.5 \%$ & 2.12 & 2.17 & 2.22 & 2.25 & 2.29 & 2.32 & 2.33 & 2.34 & 2.36 & 2.37 & 2.38 \\
\hline & & GPD 50\% & 2.15 & 2.21 & 2.28 & 2.33 & 2.39 & 2.44 & 2.46 & 2.48 & 2.53 & 2.57 & 2.61 \\
\hline & & GPD 97.5\% & 2.19 & 2.25 & 2.33 & 2.41 & 2.53 & 2.64 & 2.71 & 2.76 & 2.95 & 3.10 & 3.27 \\
\hline \multirow[t]{6}{*}{28} & Anawhata & SSJPM $2.5 \%$ & 1.86 & 1.90 & 1.95 & 1.99 & 2.04 & 2.07 & 2.09 & 2.10 & 2.14 & 2.16 & 2.18 \\
\hline & & SSJPM 50\% & 1.86 & 1.91 & 1.96 & 2.00 & 2.05 & 2.09 & 2.11 & 2.12 & 2.16 & 2.19 & 2.22 \\
\hline & & \begin{tabular}{|l|} 
SSJPM \\
$97.5 \%$
\end{tabular} & 1.87 & 1.92 & 1.98 & 2.02 & 2.07 & 2.12 & 2.14 & 2.16 & 2.21 & 2.25 & 2.29 \\
\hline & & GPD $2.5 \%$ & 1.78 & 1.83 & 1.89 & 1.92 & 1.95 & 1.97 & 1.98 & 1.99 & 2.01 & 2.02 & 2.03 \\
\hline & & GPD 50\% & 1.82 & 1.89 & 1.97 & 2.04 & 2.11 & 2.17 & 2.20 & 2.23 & 2.30 & 2.35 & 2.40 \\
\hline & & GPD $97.5 \%$ & 1.87 & 1.94 & 2.06 & 2.16 & 2.31 & 2.44 & 2.53 & 2.59 & 2.83 & 3.03 & 3.25 \\
\hline \multirow[t]{6}{*}{29} & Helensville & SSJPM 2.5\% & 2.33 & 2.39 & 2.47 & 2.53 & 2.60 & 2.65 & 2.68 & 2.70 & 2.76 & 2.80 & 2.85 \\
\hline & & SSJPM 50\% & 2.35 & 2.42 & 2.51 & 2.58 & 2.69 & 2.77 & 2.82 & 2.86 & 2.98 & 3.07 & 3.17 \\
\hline & & \begin{tabular}{|l|} 
SSJPM \\
$97.5 \%$
\end{tabular} & 2.37 & 2.45 & 2.57 & 2.68 & 2.87 & 3.05 & 3.16 & 3.25 & 3.58 & 3.88 & 4.22 \\
\hline & & GPD $2.5 \%$ & 2.21 & 2.27 & 2.34 & 2.38 & 2.43 & 2.46 & 2.48 & 2.49 & 2.52 & 2.53 & 2.55 \\
\hline & & GPD 50\% & 2.27 & 2.34 & 2.45 & 2.52 & 2.63 & 2.71 & 2.76 & 2.80 & 2.92 & 3.01 & 3.10 \\
\hline & & GPD $97.5 \%$ & 2.33 & 2.41 & 2.58 & 2.79 & 3.21 & 3.64 & 4.00 & 4.25 & 5.45 & 6.64 & 8.33 \\
\hline \multirow[t]{6}{*}{30} & Pouto Point & SSJPM $2.5 \%$ & 2.00 & 2.06 & 2.12 & 2.17 & 2.22 & 2.26 & 2.28 & 2.29 & 2.33 & 2.36 & 2.38 \\
\hline & & SSJPM 50\% & 2.02 & 2.07 & 2.14 & 2.19 & 2.24 & 2.28 & 2.30 & 2.32 & 2.36 & 2.39 & 2.42 \\
\hline & & \begin{tabular}{|l|} 
SSJPM \\
$97.5 \%$
\end{tabular} & 2.03 & 2.09 & 2.16 & 2.21 & 2.26 & 2.31 & 2.34 & 2.36 & 2.42 & 2.47 & 2.52 \\
\hline & & GPD $2.5 \%$ & 1.88 & 1.93 & 1.99 & 2.03 & 2.07 & 2.10 & 2.11 & 2.12 & 2.14 & 2.15 & 2.16 \\
\hline & & GPD 50\% & 1.92 & 1.98 & 2.07 & 2.14 & 2.22 & 2.27 & 2.31 & 2.33 & 2.40 & 2.45 & 2.49 \\
\hline & & GPD 97.5\% & 1.96 & 2.04 & 2.15 & 2.25 & 2.42 & 2.57 & 2.67 & 2.74 & 3.02 & 3.25 & 3.51 \\
\hline
\end{tabular}




\begin{tabular}{|c|c|c|c|c|c|c|c|c|c|c|c|c|}
\hline \multirow{2}{*}{ 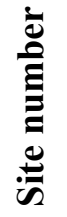 } & \multirow{2}{*}{ 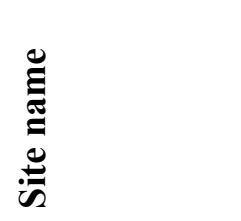 } & \multicolumn{11}{|c|}{ Return period (years) } \\
\hline & & 1 & 2 & 5 & 10 & 25 & 50 & 75 & 100 & 250 & 500 & 1000 \\
\hline 1 & Rangaunu & 0.41 & 0.46 & 0.52 & 0.56 & 0.61 & 0.64 & 0.66 & 0.67 & 0.70 & 0.73 & 0.75 \\
\hline 2 & Whangaroa & 0.32 & 0.36 & 0.40 & 0.43 & 0.47 & 0.49 & 0.50 & 0.50 & 0.53 & 0.54 & 0.55 \\
\hline 3 & Opua & 0.31 & 0.34 & 0.39 & 0.42 & 0.45 & 0.48 & 0.49 & 0.50 & 0.53 & 0.54 & 0.56 \\
\hline 4 & Marsden Point & 0.35 & 0.40 & 0.47 & 0.52 & 0.59 & 0.63 & 0.66 & 0.68 & 0.73 & 0.77 & 0.81 \\
\hline 5 & Auckland & 0.36 & 0.41 & 0.48 & 0.53 & 0.61 & 0.67 & 0.70 & 0.73 & 0.81 & 0.87 & 0.94 \\
\hline 6 & Whitianga & 0.32 & 0.36 & 0.42 & 0.46 & 0.51 & 0.55 & 0.57 & 0.59 & 0.64 & 0.67 & 0.71 \\
\hline 7 & Tararu & 0.39 & 0.43 & 0.49 & 0.53 & 0.58 & 0.62 & 0.64 & 0.66 & 0.71 & 0.74 & 0.77 \\
\hline 8 & Tauranga & 0.30 & 0.34 & 0.38 & 0.42 & 0.46 & 0.49 & 0.51 & 0.52 & 0.56 & 0.59 & 0.61 \\
\hline 9 & Moturiki & 0.29 & 0.33 & 0.39 & 0.44 & 0.52 & 0.58 & 0.62 & 0.64 & 0.74 & 0.81 & 0.90 \\
\hline 10 & Gisborne & 0.33 & 0.36 & 0.38 & 0.40 & 0.41 & 0.42 & 0.42 & 0.43 & 0.43 & 0.43 & 0.44 \\
\hline 11 & Wellington & 0.35 & 0.38 & 0.43 & 0.46 & 0.49 & 0.52 & 0.54 & 0.55 & 0.58 & 0.60 & 0.62 \\
\hline 12 & Kaikoura & 0.29 & 0.32 & 0.35 & 0.39 & 0.43 & 0.47 & 0.49 & 0.50 & 0.56 & 0.60 & 0.65 \\
\hline 13 & Sumner & 0.41 & 0.44 & 0.48 & 0.50 & 0.53 & 0.54 & 0.55 & 0.55 & 0.57 & 0.58 & 0.58 \\
\hline 14 & Lyttelton & 0.43 & 0.47 & 0.51 & 0.54 & 0.58 & 0.61 & 0.62 & 0.64 & 0.66 & 0.69 & 0.70 \\
\hline 15 & Timaru & 0.40 & 0.43 & 0.48 & 0.51 & 0.55 & 0.58 & 0.59 & 0.60 & 0.63 & 0.65 & 0.67 \\
\hline 16 & Dunedin & 0.38 & 0.42 & 0.46 & 0.49 & 0.53 & 0.55 & 0.56 & 0.57 & 0.60 & 0.62 & 0.63 \\
\hline 17 & Green Island & 0.38 & 0.41 & 0.45 & 0.47 & 0.50 & 0.51 & 0.52 & 0.53 & 0.55 & 0.56 & 0.57 \\
\hline 18 & Invercargill & 0.75 & 0.82 & 0.90 & 0.97 & 1.04 & 1.09 & 1.11 & 1.13 & 1.18 & 1.22 & 1.25 \\
\hline 19 & Dog Island & 0.42 & 0.45 & 0.49 & 0.52 & 0.55 & 0.57 & 0.58 & 0.59 & 0.61 & 0.63 & 0.64 \\
\hline 20 & Jackson Bay & 0.44 & 0.49 & 0.55 & 0.59 & 0.64 & 0.68 & 0.71 & 0.72 & 0.77 & 0.81 & 0.84 \\
\hline 21 & Charleston & 0.47 & 0.52 & 0.60 & 0.67 & 0.75 & 0.82 & 0.86 & 0.88 & 0.98 & 1.05 & 1.12 \\
\hline 22 & Nelson & 0.39 & 0.44 & 0.51 & 0.57 & 0.65 & 0.70 & 0.74 & 0.76 & 0.84 & 0.90 & 0.95 \\
\hline 23 & Kapiti & 0.38 & 0.41 & 0.45 & 0.47 & 0.50 & 0.52 & 0.53 & 0.53 & 0.55 & 0.56 & 0.57 \\
\hline 24 & Taranaki & 0.39 & 0.42 & 0.46 & 0.48 & 0.51 & 0.53 & 0.53 & 0.54 & 0.55 & 0.56 & 0.57 \\
\hline
\end{tabular}




\begin{tabular}{|l|l|l|l|l|l|l|l|l|l|l|l|l|}
\hline 25 & Kawhia & 0.59 & 0.66 & 0.77 & 0.85 & 0.95 & 1.02 & 1.06 & 1.09 & 1.18 & 1.25 & 1.31 \\
\hline 26 & Raglan & 0.58 & 0.68 & 0.83 & 0.95 & 1.15 & 1.31 & 1.41 & 1.49 & 1.76 & 1.99 & 2.25 \\
\hline 27 & Onehunga & 0.47 & 0.51 & 0.57 & 0.60 & 0.65 & 0.68 & 0.69 & 0.70 & 0.73 & 0.75 & 0.77 \\
\hline 28 & Anawhata & 0.40 & 0.44 & 0.49 & 0.52 & 0.56 & 0.58 & 0.59 & 0.60 & 0.63 & 0.64 & 0.66 \\
\hline 29 & Helensville & 0.58 & 0.65 & 0.76 & 0.83 & 0.92 & 0.99 & 1.03 & 1.05 & 1.13 & 1.18 & 1.23 \\
\hline 30 & Pouto Point & 0.43 & 0.48 & 0.54 & 0.60 & 0.67 & 0.72 & 0.75 & 0.77 & 0.84 & 0.90 & 0.95 \\
\hline
\end{tabular}


Table S9. Maximum observed water level, tide and skew-surge, and "maximum potential” water level = tide + skew-surge (m).

\begin{tabular}{|c|c|c|c|c|c|c|c|c|c|c|c|c|c|c|c|}
\hline 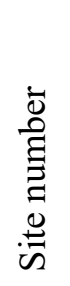 & $\begin{array}{l}\underset{\Xi}{\Xi} \\
\stackrel{\Xi}{\Xi} \\
\stackrel{\Xi}{\Xi}\end{array}$ & 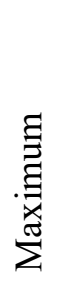 & 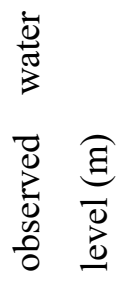 & 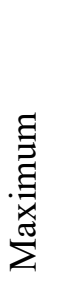 & $\begin{array}{l}: 0 \\
D \\
D \\
D \\
D \\
0 \\
0\end{array}$ & છ્છ & $\begin{array}{l}\text { 志 } \\
\text { 离 } \\
\text { 总 }\end{array}$ & 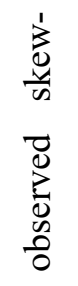 & 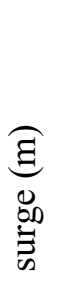 & 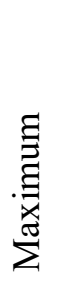 & $\begin{array}{l}+ \\
0 \\
.0 \\
0 \\
0 \\
己 \\
0 \\
0 \\
0\end{array}$ & 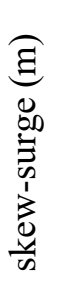 & 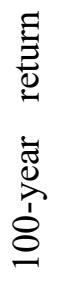 & 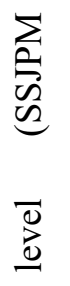 & 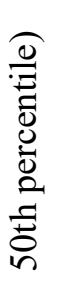 \\
\hline 1 & Rangaunu & & 1.67 & & 1.41 & & & 0.69 & & & 2.10 & & & 1.81 & \\
\hline 2 & Whangaroa & & 1.63 & & 1.29 & & & 0.50 & & & 1.78 & & & 1.65 & \\
\hline 3 & Opua & & 1.64 & & 1.34 & & & 0.44 & & & 1.78 & & & 1.65 & \\
\hline 4 & Marsden Point & & 1.71 & & 1.43 & & & 0.63 & & & 2.05 & & & 1.83 & \\
\hline 5 & Auckland & & 2.38 & & 1.89 & & & 0.75 & & & 2.64 & & & 2.22 & \\
\hline 6 & Whitianga & & 1.47 & & 1.05 & & & 0.56 & & & 1.61 & & & 1.41 & \\
\hline 7 & Tararu & & 2.68 & & 1.93 & & & 0.62 & & & 2.56 & & & 2.37 & \\
\hline 8 & Tauranga & & 1.43 & & 1.07 & & & 0.47 & & & 1.54 & & & 1.39 & \\
\hline 9 & Moturiki & & 1.59 & & 1.14 & & & 0.59 & & & 1.74 & & & 1.48 & \\
\hline 10 & Gisborne & & 1.40 & & 0.95 & & & 0.43 & & & 1.39 & & & 1.29 & \\
\hline 11 & Wellington & & 1.32 & & 0.83 & & & 0.58 & & & 1.41 & & & 1.20 & \\
\hline 12 & Kaikoura & & 1.56 & & 1.07 & & & 0.39 & & & 1.45 & & & 1.35 & \\
\hline 13 & Sumner & & 1.78 & & 1.34 & & & 0.55 & & & 1.90 & & & 1.70 & \\
\hline 14 & Lyttelton & & 2.02 & & 1.39 & & & 0.64 & & & 2.03 & & & 1.78 & \\
\hline 15 & Timaru & & 1.87 & & 1.25 & & & 0.57 & & & 1.82 & & & 1.65 & \\
\hline 16 & Dunedin & & 1.76 & & 1.37 & & & 0.59 & & & 1.96 & & & 1.72 & \\
\hline 17 & Green Island & & 1.56 & & 1.22 & & & 0.50 & & & 1.72 & & & 1.57 & \\
\hline 18 & Invercargill & & 2.50 & & 1.38 & & & 1.12 & & & 2.50 & & & 2.22 & \\
\hline 19 & Dog Island & & 3.70 & & 1.44 & & & 0.57 & & & 2.01 & & & 1.82 & \\
\hline 20 & Jackson Bay & & 2.19 & & 1.41 & & & 0.69 & & & 2.10 & & & 1.84 & \\
\hline 21 & Charleston & & 2.66 & & 1.80 & & & 0.84 & & & 2.64 & & & 2.24 & \\
\hline 22 & Nelson & & 2.76 & & 2.37 & & & 0.83 & & & 3.20 & & & 2.68 & \\
\hline 23 & Kapiti & & 1.51 & & 1.11 & & & 0.54 & & & 1.66 & & & 1.47 & \\
\hline
\end{tabular}




\begin{tabular}{|l|l|l|l|l|l|l|}
\hline 24 & Taranaki & 2.49 & 2.00 & 0.54 & 2.53 & 2.33 \\
\hline 25 & Kawhia & 2.60 & 1.94 & 1.15 & 3.09 & 2.66 \\
\hline 26 & Raglan & 2.54 & 1.88 & 1.15 & 3.04 & 2.67 \\
\hline 27 & Onehunga & 2.65 & 2.16 & 0.71 & 2.87 & 2.57 \\
\hline 28 & Anawhata & 2.28 & 1.75 & 0.58 & 2.34 & 2.12 \\
\hline 29 & Helensville & 2.83 & 2.21 & 1.04 & 3.26 & 2.86 \\
\hline 30 & Pouto Point & 2.38 & 1.88 & 0.71 & 2.59 & 2.32 \\
\hline
\end{tabular}

100 
Table S10. Climate indices coincident with extreme sea-level events. $R P=$ return period, or average recurrence interval, $S O I=$ southern oscillation index, SAM = southern annular mode, $Z 1=$ Auckland - Christchurch mean sea-level pressure $(M S L P), M 1=$ Hobart Chatham Islands MSLP, M2 = Hokitika - Chatham Islands MSLP, M3 = Hobart - Hokitika MSLP. The 'Trenberth' indices Z1, M1, 105 M2, M3 (Trenberth 1976) describe monthly mean differences in mean sea-level pressure between several climate stations in the New Zealand region (in the same way that the SOI is an index of mean sea level pressure differences between the east and west Pacific). Pressure differences are directly related to wind speed (perpendicular to the orientation of the pressure difference), hence the Trenberth indices encapsulate monthly mean wind flows over New Zealand. As such, they are well correlated with some of the monthly 'Kidson' weather type and regime frequencies, which also capture wind flows and pressure patterns around New Zealand.

\begin{tabular}{|c|c|c|c|c|c|c|c|c|}
\hline $\begin{array}{l}\text { Event } \\
\#\end{array}$ & Date/Time & $\begin{array}{l}\text { RP } \\
\text { (yrs) }\end{array}$ & SOI & $\begin{array}{l}\text { AAO } \\
\text { (SAM) }\end{array}$ & $\mathrm{Z1}$ & M1 & M2 & M3 \\
\hline 57 & 15/03/1903 16:00 & 9 & 0.87 & & 45 & -15 & 54 & -69 \\
\hline 64 & $27 / 05 / 1903$ 15:00 & 7 & 0.87 & & 34 & 6 & -34 & 40 \\
\hline 37 & $27 / 07 / 1903$ 17:00 & 15 & 0.07 & & 36 & -11 & 1 & -12 \\
\hline 17 & 4/02/1904 09:00 & 45 & 1.3 & & -2 & -29 & -8 & -21 \\
\hline 28 & 14/02/1918 22:00 & 21 & 0.9 & & -19 & -62 & -55 & -7 \\
\hline 75 & 9/06/1918 15:00 & 6 & -0.33 & & 26 & 25 & 19 & 6 \\
\hline 44 & 9/07/1918 16:00 & 12 & -0.87 & & 19 & 173 & 91 & 82 \\
\hline 9 & 4/08/1921 15:00 & 69 & 0.57 & & 45 & 6 & 13 & -7 \\
\hline 60 & $18 / 06 / 1924$ 16:00 & 8 & 0.9 & & 32 & -11 & -5 & -6 \\
\hline 56 & $23 / 02 / 192502: 00$ & 9 & 1.13 & & 25 & -17 & -26 & 9 \\
\hline 41 & 11/07/1926 20:00 & 12 & -0.67 & & -5 & -41 & -4 & -37 \\
\hline 6 & 26/03/1936 09:00 & 131 & 0.83 & & -1 & 78 & 48 & 30 \\
\hline 72 & $21 / 04 / 1936$ 15:00 & 6 & 1 & & 4 & -58 & -38 & -20 \\
\hline 74 & $30 / 06 / 193821: 00$ & 6 & 1.63 & & -4 & -37 & -31 & -6 \\
\hline 51 & 27/07/1938 19:00 & 10 & 1.6 & & -64 & 72 & -1 & 73 \\
\hline 31 & 15/08/1939 15:00 & 17 & -0.17 & & 10 & -17 & 2 & -19 \\
\hline 20 & 27/01/1940 04:00 & 35 & -0.57 & & 22 & -32 & -23 & -9 \\
\hline 54 & 20/06/1947 21:00 & 10 & -0.1 & & 28 & 13 & 21 & -8 \\
\hline 83 & 4/12/1951 08:00 & 5 & -0.67 & & 10 & 86 & 50 & 36 \\
\hline 66 & $8 / 08 / 195222: 00$ & 6 & -0.2 & & -60 & -59 & -60 & 1 \\
\hline 45 & 7/03/1954 09:00 & 12 & 0 & & 0 & -83 & -44 & -39 \\
\hline 73 & 31/12/1955 09:00 & 6 & 1.2 & & -36 & -148 & 15 & -163 \\
\hline 63 & 14/05/1956 19:00 & 7 & 1.43 & & -63 & -96 & -77 & -19 \\
\hline 50 & 14/07/1956 00:00 & 11 & 1.17 & & -10 & 27 & 17 & 10 \\
\hline 55 & $21 / 10 / 1957$ 13:00 & 10 & -0.93 & & 37 & 36 & 1 & 35 \\
\hline 30 & 27/04/1959 19:00 & 18 & 0.47 & & -2 & 48 & 22 & 26 \\
\hline 15 & 13/06/1968 22:00 & 48 & 1.13 & & -6 & 11 & -26 & 37 \\
\hline 78 & 11/07/1971 22:00 & 6 & 0.6 & & -44 & -41 & -13 & -28 \\
\hline
\end{tabular}




\begin{tabular}{|c|c|c|c|c|c|c|c|c|}
\hline $\begin{array}{l}\text { Event } \\
\#\end{array}$ & Date/Time & $\begin{array}{l}\text { RP } \\
\text { (yrs) }\end{array}$ & SOI & $\begin{array}{l}\text { AAO } \\
\text { (SAM) }\end{array}$ & $\mathrm{Z1}$ & M1 & M2 & M3 \\
\hline 76 & \begin{tabular}{|c|}
$9 / 10 / 197107: 00$ \\
\end{tabular} & 6 & 1.3 & & 19 & -7 & 20 & -27 \\
\hline 7 & 17/04/1972 19:00 & 87 & -0.77 & & 22 & 93 & 36 & 57 \\
\hline 58 & $16 / 05 / 1972$ 19:00 & 9 & -1.23 & & 5 & 112 & 28 & 84 \\
\hline 48 & $21 / 06 / 1974$ 16:00 & 11 & 0.83 & & -35 & -22 & 2 & -24 \\
\hline 27 & 14/06/1975 23:00 & 22 & 1.4 & & -18 & 49 & 18 & 31 \\
\hline 53 & 18/04/1976 23:00 & 10 & 0.53 & & -32 & -30 & -25 & -5 \\
\hline 36 & 24/05/1978 21:00 & 15 & 0.4 & & -38 & -41 & -3 & -38 \\
\hline 26 & 22/06/1978 20:00 & 23 & 0.9 & & -62 & 60 & 14 & 46 \\
\hline 25 & $20 / 07 / 197806: 00$ & 23 & 0.37 & & 0 & -18 & -17 & -1 \\
\hline 12 & $16 / 03 / 198015: 00$ & 63 & -0.93 & -1.424 & -3 & 3 & -6 & 9 \\
\hline 49 & $26 / 09 / 198423: 00$ & 11 & -0.13 & 0.327 & -33 & -65 & -28 & -37 \\
\hline 43 & 14/07/1987 23:00 & 12 & -1.97 & -1.531 & -25 & 43 & 20 & 23 \\
\hline 33 & 8/02/1989 11:00 & 16 & 0.93 & 0.849 & -8 & 42 & -12 & 54 \\
\hline 82 & $23 / 08 / 198900: 00$ & 5 & 0.17 & -0.132 & -46 & -70 & -45 & -25 \\
\hline 39 & 25/04/1990 07:00 & 14 & 0 & -1.879 & 39 & 21 & 19 & 2 \\
\hline 24 & 15/06/1991 22:00 & 27 & -0.97 & -1.155 & -3 & 74 & 61 & 13 \\
\hline 59 & 19/09/1993 22:00 & 9 & -1.4 & 1.061 & -15 & 80 & 35 & 45 \\
\hline 23 & 14/07/1995 20:00 & 29 & 0 & -3.01 & 41 & 62 & -5 & 67 \\
\hline 52 & 2/08/1996 22:00 & 10 & 0.53 & -1.502 & 10 & 61 & 36 & 25 \\
\hline 8 & 11/03/1997 22:00 & 84 & -0.63 & 0.701 & -29 & 15 & 4 & 11 \\
\hline 11 & 30/03/1998 03:00 & 65 & -2.83 & 0.736 & 41 & -14 & -2 & -12 \\
\hline 5 & 17/04/1999 10:00 & 249 & 1 & 0.949 & -21 & 39 & -17 & 56 \\
\hline 2 & 15/06/1999 18:00 & 942 & 0.2 & -1.325 & -2 & 41 & 18 & 23 \\
\hline 69 & 3/06/2000 23:00 & 6 & -0.23 & 0.117 & 27 & -4 & -15 & 11 \\
\hline 22 & $3 / 07 / 200021: 00$ & 30 & -0.23 & 0.059 & -27 & -104 & -44 & -60 \\
\hline 21 & $30 / 08 / 200020: 00$ & 32 & 0.3 & -0.674 & -12 & 31 & 7 & 24 \\
\hline 67 & 12/01/2001 09:00 & 6 & 0.93 & -0.471 & -3 & 64 & 30 & 34 \\
\hline 81 & $13 / 04 / 2001$ 10:00 & 5 & -0.13 & 0.515 & -6 & -35 & -2 & -33 \\
\hline 40 & 19/08/2001 16:00 & 14 & -0.43 & 0.91 & 9 & -24 & -10 & -14 \\
\hline 47 & $30 / 03 / 2002$ 17:00 & 12 & -0.23 & -1.823 & 35 & 41 & 3 & 38 \\
\hline 46 & 24/05/2002 02:00 & 12 & -0.93 & -2.798 & 24 & 87 & 25 & 62 \\
\hline 16 & \begin{tabular}{|l|}
$16 / 04 / 2003 ~ 18: 00$ \\
\end{tabular} & 46 & -0.8 & 0.224 & -42 & 58 & 20 & 38 \\
\hline 70 & \begin{tabular}{|l|}
$26 / 12 / 200306: 00$ \\
\end{tabular} & 6 & -0.3 & -1.323 & 15 & -1 & 9 & -10 \\
\hline 29 & $18 / 09 / 200523: 00$ & 19 & 0.13 & 0.241 & 3 & -1 & 10 & -11 \\
\hline
\end{tabular}




\begin{tabular}{|c|c|c|c|c|c|c|c|c|}
\hline $\begin{array}{l}\text { Event } \\
\#\end{array}$ & Date/Time & $\begin{array}{l}\text { RP } \\
\text { (yrs) }\end{array}$ & SOI & $\begin{array}{l}\text { AAO } \\
\text { (SAM) }\end{array}$ & $\mathrm{Z1}$ & M1 & M2 & M3 \\
\hline 42 & $3 / 01 / 200603: 00$ & 12 & 0.37 & 0.339 & 12 & 13 & 2 & 11 \\
\hline 38 & $7 / 06 / 200820: 00$ & 14 & 0.03 & 1.348 & 23 & 20 & 15 & 5 \\
\hline 34 & 27/07/2008 01:00 & 15 & 0.47 & 0.32 & 5 & 13 & -15 & 28 \\
\hline 77 & $30 / 07 / 200822: 00$ & 6 & 0.47 & 0.32 & 5 & 13 & -15 & 28 \\
\hline 71 & 13/12/2008 06:00 & 6 & 1.37 & 1.194 & -8 & -37 & -20 & -17 \\
\hline 13 & $23 / 07 / 200923: 00$ & 62 & -0.3 & -1.234 & -1 & 14 & 15 & -1 \\
\hline 61 & $1 / 03 / 201014: 00$ & 8 & -1.6 & 0.108 & 24 & 33 & 29 & 4 \\
\hline 19 & 13/08/2010 01:00 & 35 & 2.13 & 1.51 & -2 & -26 & -21 & -5 \\
\hline 4 & $23 / 01 / 201110: 00$ & 330 & 2.4 & 0.052 & -24 & -12 & -1 & -11 \\
\hline 85 & $11 / 05 / 201222: 00$ & 5 & -0.77 & 0.153 & -16 & -14 & 6 & -20 \\
\hline 65 & $5 / 06 / 201223: 00$ & 7 & -0.57 & -0.197 & 26 & 52 & 28 & 24 \\
\hline 79 & $6 / 05 / 201307: 00$ & 6 & 0.87 & 0.36 & -25 & 34 & -6 & 40 \\
\hline 14 & $26 / 05 / 201314: 00$ & 50 & 0.87 & 0.36 & -25 & 34 & -6 & 40 \\
\hline 32 & $21 / 06 / 201301: 00$ & 16 & 1.11 & -0.271 & -21 & 38 & -10 & 48 \\
\hline 68 & $24 / 09 / 201322: 00$ & 6 & 0.05 & -1.658 & -1 & -6 & 6 & -12 \\
\hline 18 & 11/03/2016 03:00 & 37 & -1.34 & 2.038 & & & & \\
\hline 84 & 17/11/2016 09:00 & 5 & -0.13 & -1.508 & & & & \\
\hline 80 & $30 / 04 / 201720: 00$ & 5 & 0.09 & 0.619 & & & & \\
\hline 10 & $21 / 07 / 201713: 00$ & 67 & 0.1 & 0.728 & & & & \\
\hline 3 & $5 / 01 / 201810: 00$ & 770 & 0.04 & 1.275 & & & & \\
\hline 1 & 1/02/2018 11:00 & 1000 & 0.48 & 1.041 & & & & \\
\hline 62 & $22 / 05 / 201823: 00$ & 8 & 0.13 & -0.077 & & & & \\
\hline 35 & 15/08/2018 01:00 & 15 & -0.5 & -0.343 & & & & \\
\hline
\end{tabular}

